INRA Prod. Anim., 2011, 24 (1), 113-128

\title{
Conception et évaluation de systèmes d'élevage durables en régions chaudes
}

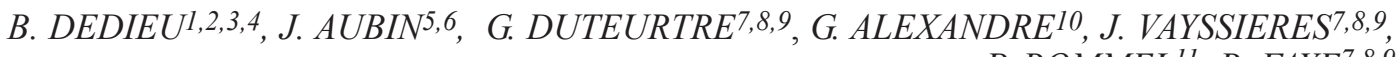
P. BOMMEL ${ }^{11}$, B. FAYE $7,8,9$

Avec la collaboration de M. MAHIEU ${ }^{10}$, A. FANCHONE 10, J.-F. TOURRAND ${ }^{10}$, A. ICKOWICZ7,8,9

${ }^{1}$ INRA, UMR1273 Mutations des Activités, des Espaces et des Formes d'Organisation dans les Territoires Ruraux, F-63122 Saint-Genès-Champanelle, France

2 AgroParisTech, Mutations des Activités, des Espaces et des Formes d'Organisation dans les Territoires Ruraux, 16 rue Claude Bernard, F-75231 Paris, France

${ }^{3}$ CEMAGREF, Mutations des Activités, des Espaces et des Formes d'Organisation dans les Territoires Ruraux, 24 avenue des Landais, F-63172 Aubière, France

${ }^{4}$ VetAgroSup, Mutations des Activités, des Espaces et des Formes d'Organisation dans les Territoires Ruraux, Site de Marmilhat, F-63370 Lempdes, France

5 INRA, UMR1069 Sol Agro et hydrosystème Spatialisation, F-35042 Rennes, France

6 Agrocampus-Ouest, Sol Agro et hydrosystème Spatialisation, F-35042 Rennes, France

${ }^{7}$ INRA, UMR0868 Systèmes d'Elevage Méditerranéens et Tropicaux, 2 place Viala, F-34060 Montpellier, France 8 CIRAD, Systèmes d'Elevage Méditerranéens et Tropicaux, Campus International de Baillarguet, F-34398 Montpellier, France

${ }^{9}$ Supagro, Systèmes d'Elevage Méditerranéens et Tropicaux, 2 place Viala, F-34060 Montpellier, France 10 INRA, UR0143 Unité de Recherches Zootechniques, Domaine Duclos Prise d'eau, F- 97170 Petit-Bourg, Guadeloupe

11 CIRAD, UR GREEN, Campus international de Baillarguet, F-34398 Montpellier, France Courriel : benoit.dedieu@clermont.inra.fr

Les enjeux liés au changement climatique et à la sécurité alimentaire confortent la nécessité de mettre au point des démarches de conception/évaluation de systèmes durables, qu'il s'agisse d'améliorer les situations existantes ou d'imaginer de nouvelles voies de développement. En régions chaudes, l'élevage remplit aussi des fonctions non productives et doit s'adapter aux aléas et incertitudes.

L'élevage est au cœur des débats sur la capacité de l'agriculture à répondre aux défis globaux de l'alimentation, de la limitation des gaz à effet de serre (GES) et de la consommation d'énergie fossile et enfin de la préservation de la biodiversité (Steinfeld et al 2006, Coulon et Lecomte 2009). Mais l'élevage est aussi un acteur essentiel du développement de nombreux territoires ruraux (Manoli et al 2010), parce qu'il conditionne l'utilisation des espaces agricoles et les paysages, mais aussi parce qu'il marque les cultures humaines, les dynamiques économiques et sociales par les multiples fonctions (productives ou non) qu'il remplit auprès des sociétés rurales (Kruska et al 2003, Gibon et Ickowicz 2010). Enfin, l'élevage est une activité humaine, qui demeure dans la très grande majorité des cas liée aux trajectoires de vie des familles (nucléaires ou élargies), intervenant fortement dans leur rapport à l'environnement, dans leurs capacités d'adaptation, dans l'organisation sociale locale, et dans les mécanismes d'accumulation (Dedieu et al 2008b, Duteurtre et Faye 2009).

Les régions chaudes méritent en soi l'attention de la recherche, compte tenu de la spécificité des situations rencontrées et ce d'autant plus qu'elles correspondent pour l'essentiel à des pays en voie de développement ou émergents ainsi qu'à des territoires «périphériques» de pays développés. Les questions de sécurité alimentaire y sont et seront particulièrement aiguës dans les années à venir (INRA-CIRAD 2009). Les pressions s'accroissent sur la capacité des sociétés rurales à fournir des produits alimentaires pour les popula- tions, dans un contexte d'urbanisation forte, tout en limitant les impacts négatifs sur l'environnement, notamment la destruction des forêts tropicales, la fragilisation des biomes sensibles, l'assèchement des ressources en eau, ainsi que l'excès de rejets de nutriments dans des zones à fortes concentrations d'élevage (feedlots, élevages industriels de volailles ou de porcs). L'enjeu alimentaire ne porte pas uniquement sur l'accroissement de la fourniture en protéines animales, mais aussi sur certains produits animaux spécifiques. L'élévation du niveau de vie est un facteur de changement des habitudes alimentaires avec un déplacement de la consommation vers certains produits animaux à forte charge symbolique (mode alimentaire plus occidental) ou culturelle (fêtes religieuses). Ainsi au Vietnam, la consommation de lait par habitant 
augmente rapidement de façon corrélée au PIB/habitant (Suzuki et al 2006). Enfin, les modèles climatiques laissent augurer, d'ici la fin du siècle, une diminution de la pluviométrie dans les régions déjà semi-arides ou à climat méditerranéen (Sciortino 2010). Dans cette perspective, l'élevage en régions chaudes sera sans doute particulièrement affecté par le changement climatique. Tous ces éléments militent pour une réflexion de fond sur les modèles d'élevage, sur la conception de systèmes durables, à la fois pour les agriculteurs, leurs familles et les sociétés rurales, mais aussi en termes de contribution au développement des territoires du Sud. Enfin, parce qu'elles témoignent de contextes exacerbés vis-à-vis de conditions d'élevage européen, les régions chaudes peuvent également nous aider à repenser nos façons d'analyser et de concevoir des systèmes d'avenir. La phase d'incertitude politique (réforme de la PAC), d'instabilité des prix et des marchés, et de plus grande fréquence d'évènements climatiques extrêmes, dans laquelle est entré l'élevage européen, n'est que le reflet modéré des conditions incertaines auxquelles bon nombre d'éleveurs du Sud ont l'habitude de faire face.

Nous proposons dans cet article de discuter des démarches de conception et d'évaluation de systèmes d'élevage durables en régions chaudes, en nous appuyant sur des travaux réalisés par l'INRA et le CIRAD. La conception de systèmes d'élevage durables intègre peu ou prou les enjeux généraux présentés cidessus dans des domaines d'application qui prennent en compte les spécificités de systèmes d'élevage locaux. Nous verrons dans la première partie du texte quels peuvent être les points remarquables et les éléments de diversité des systèmes d'élevage des régions chaudes. $\mathrm{La}$ conception peut être «réglée», c'est-àdire qu'elle vise l'amélioration de situations existantes en vue de satisfaire de nouveaux objectifs, ou «innovante» lorsqu'elle vise des situations nouvelles vers lesquelles on veut tendre, mais avec des objectifs plus flous, des cadres d'analyse plus incertains et des connaissances disponibles plus rares (Meynard et al 2006). L'évaluation participe au processus de conception en fixant des repères à atteindre ou en balisant une direction à suivre. La définition des principes, critères et indicateurs d'évaluation de la durabilité fait appel à une expertise globale scientifique (élevage et GES par exemple), mais aussi à des débats locaux entre acteurs des territoires (ce qu'ils attendent de l'élevage dans l'avenir, Rey-Valette et al 2008) et avec les éleveurs (comment améliorer le fonctionnement de leurs exploitations, relativement à leurs attentes). La deuxième partie du texte propose quelques éléments de réflexion générale sur les démarches de conception/évaluation et les questions que posent l'application de ces démarches dans les régions chaudes. Dans une troisième partie, nous illustrons des démarches de conception à partir d'exemples issus de travaux récents s'appuyant sur le développement de modèles de systèmes d'élevage. Dans une dernière partie, nous mettons en perspective les points essentiels pour le développement de démarche de conception/évaluation de systèmes en référence à la durabilité.

\section{1 / Spécificités et diversité des systèmes d'élevage en régions chaudes}

L'élevage en régions tropicales et méditerranéennes présente plusieurs spécificités. La plus évidente renvoie à la nature des contraintes écologiques qui s'exercent sur ces systèmes. Ainsi, les espèces élevées et les conduites d'élevage constituent des adaptations à des conditions climatiques et pastorales caractérisées comme «difficiles», qu'il s'agisse de milieu équatoriaux à forte pression sanitaire, de zones sèches à forte contrainte hydrique, ou de zones marquées par des fortes irrégularités climatiques saisonnières.

Mais les facteurs écologiques ne sont pas les seuls à prendre en compte : il faut aussi considérer les fonctions multiples des animaux au sein des sociétés d'éleveurs. L'élevage joue un rôle majeur, bien au-delà de sa fonction de production de biens, dans des sociétés rurales souvent vulnérables, sans filet de sécurité. Celles-ci doivent en effet faire face à différents types de risques et d'incertitudes, de nature climatique mais aussi sanitaire, politique et marchande (Duteurtre et Faye 2009). La multifonctionnalité de l'élevage est ainsi une première caractéristique essentielle des systèmes des régions chaudes, et l'appréciation des fonctions dominantes constitue une clé de différenciation des systèmes. Nous y revenons dans la section 1.1. En second lieu, les modes d'élevage sont très dépendants de la disponibilité des ressources alimentaires. La mobilité est un des leviers de la flexibilité vis-àvis des aléas climatiques et de disponibilité alimentaire (Noziere et al 2011), mais l'association culture-élevage pourvoie également à l'alimentation des troupeaux. Il tend aussi à se rapprocher des bassins de consommation contribuant au fort développement d'un élevage périurbain (Guerin et Faye 1999). Ce rapport aux ressources constitue une autre clé de différenciation des systèmes techniques que nous présentons dans la section 1.2.

\section{1 / Multifonctionnalité de l'élevage en régions chaudes}

La gestion des risques divers et fréquents auxquels sont soumises les sociétés rurales dans les régions chaudes qui hébergent la plupart des pays les moins avancés, s'appuie en grande partie sur l'élevage, faute de disposer de services, d'assurances ou d'appuis institutionnels suffisants. L'activité d'élevage joue ainsi un rôle majeur de sécurisation des familles, rôle d'épargne, d'assurance sociale, de capital mobilisable tant sur le plan social qu'économique, en même temps qu'il est pourvoyeur de produits et gestionnaire d'un milieu incertain et variable (Ancey et al 2009). Par ailleurs, dans les régions chaudes, les populations sont beaucoup plus nombreuses à conserver un mode de vie rural que dans les pays tempérés plus développés. Or, l'élevage qui constitue à la fois un des piliers des économies pastorales et agropastorales, est aussi un élément central des trajectoires d'accumulation, de diversification et d'intensification des systèmes agricoles (Duteurtre et Faye 2009). Du coup, ces systèmes d'élevage intègrent d'importants enjeux économiques et sociaux, audelà même de ce qui a trait directement à la production animale. L'animal, en tant que capital familial, mais aussi comme source d'épargne, de revenus, et comme facteur de production (animale, de travail, de fumure) constitue ainsi un bien précieux, plus précieux souvent que dans les contextes intensifs des pays tempérés (voir dans ce même numéro Alary et al 2011).

Ces caractéristiques générales masquent une diversité des situations, à l'échelle des grandes régions tropicales et méditerranéennes, mais aussi au niveau plus local. Par exemple, Alary et al (2007) différencient ainsi plusieurs types d'éleveurs laitiers dans le bassin de Mbarara (Ouganda) selon les fonctions de l'élevage et les stratégies commerciales associées (encadré 1). D'autres critères de différenciation ont été mis en évidence dans les élevages Antillais tant porcins que de ruminants (Zebus et al 2005, Diman et al 2006, Alexandre et al 2008). L'opposition entre la filière patrimoniale et la filière productiviste structure ainsi la représentation de la diversité des systèmes en Guadeloupe. On différencie ainsi un élevage à fonction sociale (d'emploi de main-d'œuvre dans un département à chômage endémique) ou de valorisation des sous-produits et de production de fumier, d'un élevage construit sur le modèle européen visant une productivité élevée. 
Encadré 1. Diversité des stratégies d'élevage bovin laitier dans le bassin de Mbarara (Alary et al 2007).

1) Une stratégie de sécurisation pour laquelle la fonction sociale de l'élevage est prépondérante sur sa fonction économique. Les producteurs privilégient la sécurisation de leur système de production avant la «rentabilité» économique.

2) Une stratégie de vente opportuniste des surplus des produits de l'élevage en particulier le lait.

3) Une stratégie d'épargne lorsque l'élevage est une forme d'investissement servant de tremplin pour d'autres activités ou pour disposer d'un capital facilement mobilisable à court ou moyen terme.

4) Une stratégie de diversification qui se distingue de la précédente par la volonté de tirer parti de toutes les activités agricoles et d'élevage de l'exploitation, instaurant un équilibre entre les différentes sources de revenus.

5) Une stratégie d'investissement qui souvent n'est pas l'œuvre de paysans, mais plutôt de commerçants ou de fonctionnaires soucieux d'accroître leurs revenus.

6) Une stratégie entrepreneuriale, principalement le fait d'éleveurs ayant une vision «techniciste», ou bien de grandes firmes agroalimentaires.

\section{2 / Des modes d'élevage diffé- renciés selon les ressources et la localisation (rural/périurbain)}

Sur le plan fonctionnel, et pour répondre aux contraintes ou opportunités climatiques, géographiques, sociales et économiques, les éleveurs adoptent des modes d'élevage, de gestion de l'espace et des ressources, qui s'adaptent à différents types de milieu (agro-écologique et climatique ainsi que rural/périurbain) et d'association avec d'autres activités. Ainsi, la littérature décline plusieurs clés d'entrée pour décrire la diversité technique des systèmes existants :

1. Le milieu (dans ses dimensions climatiques et spatiales) conduit à orienter le type de production. Ainsi le degré d'aridité du milieu délimite la capacité de charge (systèmes plus ou moins extensifs). De même, la proximité urbaine module les systèmes d'élevage en fonction de l'accès aux marchés, aux services et surtout en fonction de l'accès au foncier; ce dernier point prend une importance accrue dans le cas des petites économies insulaires à forte pression démographique comme dans les DOM français ;

2. La mobilité : la capacité qu'ont les éleveurs de répondre aux aléas climatiques par la mobilité du cheptel est un facteur-clé de la distinction entre les modèles nomades/transhumants et les modèles sédentaires et représente sans doute le paramètre le plus discriminant. La régularité des déplacements (aléatoires ou pendulaires) est un facteur permettant de discriminer les systèmes entièrement contraints par les aléas climatiques, et ceux soumis à une différenciation spatiale et négociée sur le plan du foncier (modèle nomade $v$ s modèle transhumant);

3. Le degré de spécialisation de l'activité (principalement abordé au regard de l'association cultures-élevages) et le degré de spécialisation du troupeau qui reflètent le type de spéculation prédominant, en particulier le lait ou la vian- de, ou encore le choix des génotypes dans une même filière. L'association agriculture-élevage en régions chaudes peut prendre deux formes : celle de l'intégration, mais qui s'appuie sur une synergie permanente entre l'élevage (transfert de fertilité, traction attelée) et l'agriculture (sous-produits agricoles pour l'alimentation), et celle de la cohabitation, compatible avec la mobilité des troupeaux. Les activités agricoles conjointes peuvent être le fait d'éleveurs mobiles ou non et dès lors déboucher sur des cohabitations provisoires/ éphémères ou plus ou moins pérennes entre l'élevage et la production agricole vivrière ou de rente;

4. Le mode de conduite du troupeau (la divagation ou non des animaux, l'attache des animaux sur parcours ou sur des espaces marginaux, les pratiques d'allotement ou de complémentation, le confiage...). Les ressources destinées à l'alimentation des animaux, selon qu'elles sont naturelles, cultivées sur l'exploitation ou achetées, déterminent le degré de dépendance vis-à-vis du marché. C'est aussi le cas pour le choix des génotypes qui renvoie à un schéma endogène (adapté et intégré) ou exogène (externalisé et très soutenu, dans les DOM ou à l'occasion de plans de développement comme le très récent plan d'infusion de sang laitier européen dans les cheptels du Sénégal).

Ces critères peuvent être hiérarchisés dans une perspective de construction des «grands» systèmes d'élevage présents dans les pays tropicaux comme dans la figure 1. Cette classification basée sur la mobilité, la diversité des activités, les sources d'aliments et le milieu reprend les principaux critères de la classification mondiale des systèmes d'élevage (Sere et Steinfeld 1996) pour mettre en relief les spécificités des élevages en régions chaudes. $\mathrm{Au}$ total, on obtient une dizaine de grands systèmes dont chacun recouvre bien entendu une variété importante de possibilités.

\section{3 / Conclusion}

On voit bien que les systèmes d'élevage de régions chaudes présentent une diversité de caractéristiques qui n'a pas seulement trait aux orientations de la production animale et au degré d'intensification comme en Europe mais aussi plus en amont, aux fonctions même de l'élevage pour la sécurisation de la famille vis-à-vis d'aléas de toute nature et au rapport aux ressources, en lien avec la localisation (élevage rural et périurbain) et la mobilité. Les questions de durabilité à l'échelle de l'exploitation ne sauraient donc se limiter à la recherche de nouveaux équilibres entre production/productivités animales et préservation de l'environnement en explorant de nouvelles pratiques. Elles nécessitent d'affronter $i$ ) la complexité des liens famille-activité d'élevage, ii) le rapport qu'ont les éleveurs aux aléas et à l'incertitude (climatique, politique et de marché) qui pèsent tant sur l'activité d'élevage, que sur les cycles de vie des familles (Dedieu et Ingrand 2010), iii) l'insertion de l'élevage dans les dynamiques territoriales notamment démographiques et foncières (Ickowicz et al 2010).

\section{2 / Concevoir et évaluer des systèmes d'élevage : élé- ments généraux}

Béguin (2007) analyse l'activité (le processus) de conception de systèmes innovants selon 3 questions : i) qu'est ce qui est cristallisé dans la conception? (quelles connaissances scientifiques ou empiriques sont formalisées? quel est le statut de l'évaluation, des critères et indicateurs ?); ii) y a-t-il une plasticité dans la conception? (plusieurs options de mise en œuvre sont-elles possibles?) ; iii) comment s'opère le développement de l'innovation, c'est-à-dire les échanges, les apprentissages mutuels entre concepteurs et utilisateurs au cours du processus?

L'interaction entre l'activité humaine et les processus biotechniques de mobilisation de ressources et d'élaboration de la production animale est caractéristique du "système d'élevage» (Gibon et al 1999, Dedieu et al 2008a). Pour aborder la question de la cristallisation au cours du processus de conception, il convient d'abord de comprendre et de formaliser le fonctionnement du système, c'est-à-dire de considérer les finalités des éleveurs et leurs logiques d'action (nous y revenons dans la section 2.1), et de caractériser les 
Figure 1. Construction hiérarchisée des grands systèmes d'élevage dans les pays tropicaux à partir de 9 critères discriminants.

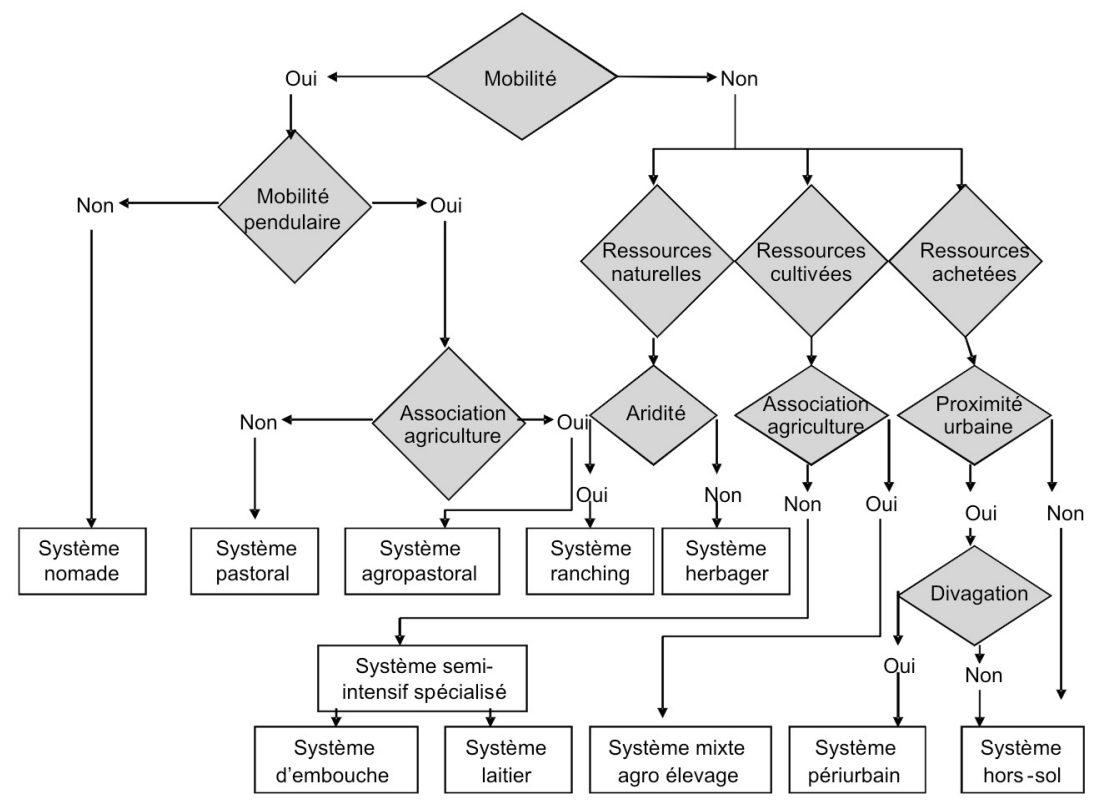

Description de quelques systèmes

- Systèmes pastoraux : il convient par exemple de distinguer les systèmes transhumants à troupeaux plurispécifiques ou monospécifiques, mobiles par réaction au climat et/ou à objectif de commercialisation de la zone sahélienne (Diop et al 2003), des systèmes s'appuyant sur une différenciation spatiale du troupeau (Ingrand et Faye 2004), la partie productive (animaux laitiers) étant sédentarisée autour des villes, la partie non-productive demeurant en zone pastorale, comme c'est le cas des systèmes camelins en Mauritanie (Faye et al 2003) ou des systèmes bovins au Niger ;

- Systèmes laitiers : il existe un fort gradient d'intensification entre des systèmes basés sur des parcours avec des races locales et des systèmes en zones climatiquement plus favorables (zones d'altitude), où l'adaptation des races exotiques est parfaitement possible, comme on peut l'observer en Ouganda ou au Vietnam (Grimaud et al 2007, Hostiou et al 2010);

- Systèmes hors-sol : souvent spécialisés, de petite taille et généralement monospécifiques (porcs, volailles, ruminants laitiers), les systèmes hors-sol peuvent avoir des degrés d'intégration avec les firmes d'aliments du bétail fort variables. A titre d'exemple, les systèmes bovins laitiers de la région de Ba-Vi au Vietnam sont liés par contrat aux laiteries privées ou publiques. Ces systèmes sont comme dans le cas de l'élevage porcin aux Antilles (Zebus et al 2004 2005) de pâles représentations d'un modèle exogène suggéré comme fer de lance d'un développement mais souvent voués à l'échec quand ils ne bénéficient pas de forts soutiens institutionnels et de subventions.

fonctionnements biotechniques. Sur ce dernier volet, nous invitons le lecteur à se reporter aux publications d'Archimède et al (2011) et Mandonnet et al (2011) dans ce numéro spécial. Mais il s'agit également de considérer les critères et indicateurs d'évaluation : nous en discutons dans la section 2.2. Enfin nous reviendrons sur les questions que posent la plasticité et le développement de l'innovation dans la section 2.3.

\section{1 / Prendre en compte les logiques d'action des éleveurs}

La connaissance des logiques d'action des éleveurs de régions chaudes a bénéficié de deux dynamiques de recherche principales: un courant en socio-anthropologie portant sur l'analyse des sociétés agricoles et pastorales et un courant hérité de l'agronomie et de la zootechnie orienté sur l'étude des systèmes d'élevage.
L'analyse des sociétés au sein desquelles est pratiqué l'élevage d'animaux domestiques dans ses différentes formes, a permis de mettre en évidence la grande diversité des communautés pratiquant l'élevage. Les chercheurs en sciences sociales ont souligné la difficulté de réduire le rôle des familles à celui d' «éleveurs» au sens strict. Dans cette optique, le pastoralisme transhumant ou nomade, l'agro-pastoralisme, l'agriculture paysanne ou d'autres formes d'élevage ont été analysés non pas comme des activités uniquement productives, mais plutôt comme des mes de vie». Ces études ont souligné les cohérences intimes qui existent entre rationalités individuelles, choix techniques, pressions foncières et environnementales, organisation sociale et rapports marchands (Bonfiglioli 1984, Blanc-Parmard et Boutrais 1994, Scoones 1999, Ancey et al 2007, Bah et «modes de production» ou des «systè- al 2010). Les recherches en zootechnie des systèmes d'élevage en régions chaudes ont abordé les individus et les groupes humains en priorité à travers leurs activités d'élevage des animaux, c'est-à-dire en étudiant les systèmes biotechniques d'élaboration des productions animales (Dedieu et al 2008a). Ces approches ont privilégié l'analyse des pratiques des éleveurs et des performances de l'élevage (Thewis et al 2005). De tels travaux ont abouti à souligner l'importance des contraintes environnementales, économiques, et sociales qui caractérisent les systèmes d'élevage en régions chaudes (Lhoste 2002).

Aujourd'hui, les apports croisés de ces deux courants permettent d'offrir une vision relativement nouvelle des systèmes d'élevage tropicaux et méditerranéens : ils apparaissent influencés localement à la fois par leur histoire socioculturelle, par les changements environnementaux et les crises sanitaires, par les mutations des marchés, par les réseaux locaux d'acteurs des territoires, et par des recompositions sociales et politiques plus globales. Dans ce cadre, les stratégies des éleveurs et les modalités de gestion du troupeau sont enchâssées dans des systèmes sociotechniques relativement complexes (Lemery 2003). La caractérisation des finalités de l'élevage et des logiques d'action des éleveurs s'avère donc délicate. Elle mérite en soi des investigations spécifiques.

Une posture négligeant l'analyse fine des logiques d'actions prendrait le risque de considérer les éleveurs du Sud en référence au seul modèle européen ou en référence à l'agent rationnel de la théorie économique standard. $\mathrm{La}$ famille agricole évoluerait dans un environnement social et politique réduisant les incertitudes radicales, ou offrant des assurances et des garanties en cas de coup dur ; l'éleveur serait le chef d'une entreprise aux contours précis ; l'atelier d'élevage serait une activité réduite à la seule fonction de production ; enfin, les pratiques et les décisions des éleveurs seraient exclusivement orientées vers la maximisation du profit. Sans évacuer l'idée que ce type de situation puisse exister dans les régions chaudes, l'encadré 2 montre, au travers d'une double approche sociologique et zootechnique réalisée dans les systèmes pastoraux du Sahel, combien la réalité est certainement à la fois plus complexe et plus diverse. Notamment, le terme «d'éleveur» apparaît parfois insuffisant pour rendre compte des identités des populations impliquées, de leurs systèmes de pensée et d'activités, et de leur relation avec le reste de la société (Wane et al 2010). Il s'agit donc d'être prudent dans l'utilisation des concepts et de la 
Encadré 2. Les logiques de gestion de troupeaux dans les systèmes pastoraux sahéliens (d'après Ancey et al 2007).

Dans un milieu aussi marqué par les aléas que le Sahel, la sécurisation des systèmes pastoraux passe à la fois par l'entretien de la solidarité familiale ainsi que par une gestion adaptative de l'activité d'élevage. Le troupeau est alors au centre de l'un et de l'autre. Sa composition, notamment l'organisation des filiations entre générations animales reflète les dons, des prêts, des alliances au sein de familles élargies et au-delà, le rôle patrimonial essentiel sur lequel se fonde la solidarité familiale. La gestion rend compte de la façon dont le chef et différents membres de la famille élargie prennent les décisions de réforme ou de vente, ainsi que d'alimentation (mobilité, complémentation). A partir d'une étude combinant un regard zootechnique (notation de l'état corporel des vaches au sortir de la saison sèche, conduite du troupeau) et sociologique (analyse des relations et représentations sociales), trois types de gestion ont ainsi été identifiées

1) La gestion centralisée, qui est le fait d'un chef de famille porteur d'une vision globale du troupeau. Cette vision globale est utile pour décider quelles vaches doivent cesser d'être traites de façon à préserver à la fois les animaux et la satisfaction des besoins familiaux. Les vaches sont en bon état corporel.

2) La gestion est collective et réalisée par plusieurs frères habitant le même campement. Ces frères ont une certaine autonomie dans la décision, par exemple dans la décision d'arrêt de traite. La multiplication des centres de décision implique une exploitation plus intensive des animaux, sans la vision globale qui caractérise le style de gestion précédent. Les vaches sont plus maigres que précédemment

3) La gestion est disjointe : le chef de famille a une activité extérieure et délègue la gestion quotidienne, en se réservant l'autorité des décisions de vente. Les revenus extérieurs permettent ces achats et autorisent une mobilité plus réduite. Les bêtes sont également en bon état, via la pratique d'une complémentation en fin de saison sèche.

nomenclature zootechnique et économique usuelle, pour être capable de concevoir des systèmes innovants adaptés aux réalités locales.

\section{2 / Les objectifs et les outils de l'évaluation des systèmes}

La question de l'évaluation des systèmes est une question complexe qui met en jeu les finalités (qui débat de son champ et de son objectif), les indicateurs et critères (quelles variables mobiliser, construire) et les démarches d'agrégation ou d'intégration des critères à l'échelle des systèmes. Nous souhaitons ici illustrer trois domaines différents de réflexion sur l'évaluation des systèmes d'élevage en régions chaudes : l'un porte sur les performances technico-économiques des systèmes, l'autre sur l'analyse environnementale, enfin le dernier sur le domaine des processus de production. Ces exemples montrent aussi la grande sensibilité des méthodologies à leur finalité (commerciale, développement).

Il existe maintenant, un grand nombre de méthodes et de cadres d'analyse du développement durable que l'on peut juger comme normatifs car reconnus par les institutions et souvent appliqués de façon standardisée sur les objets et systèmes qu'ils sont censés évaluer. On peut par exemple citer le Global Reporting Initiative, les indicateurs de l'OCDE, certaines méthodes bénéficiant de normes ISO (voir norme développement durable ISO 26000 en préparation...). La normalisation de ces modes d'évaluation garantit la répétabilité des mesures, une comparabilité des résultats quels que soient les contextes et une définition claire et partagée de leur contenu. Dans le domaine de l'agriculture, ces approches ont conduit au développement de systèmes d'indicateurs de performances ouvrant sur des démarches de certification, ou d'analyse des systèmes, voire d'auto-analyse comme c'est le cas dans la méthode IDEA (Vilain et al 2003). Les approches standardisées d'évaluation des systèmes sont donc largement répandues et ont donc été appliquées de façon plus ou moins complète aux systèmes d'élevage en régions chaudes.

\section{a) Les performances technico-écono- miques des systèmes}

Compte tenu des caractéristiques de l'élevage en régions chaudes, brièvement résumées ci-dessus, ce sont moins les performances techniques et économiques des ateliers d'élevage qu'il faut discuter (les performances apparaissent souvent assez éloignées des référentiels des zones tempérées) que la nature des référentiels de performances qui n'ont souvent pas de sens vis-à-vis des réalités locales. La définition des critères d'évaluation technico-économique d'un atelier d'élevage se réfère le plus souvent à la fonction de production de l'activité d'élevage et à la finalité de revenu de la famille au détriment des autres fonctions et finalités. Or, dans certaines régions, les schémas de développement proposés par les autorités publiques s'appuient sur cette grille d'analyse et considèrent les faibles résultats de certains systèmes «paysans» ou «traditionnels» (comparativement à des unités de production qualifiées de «professionnelles modernes» relevant d'un modèle dominant) comme l'expression d'un retard de technicité et non comme l'expression de fonctions différentes de l'élevage (Zebus et al 2005, Diman et al 2006). De ce fait, les systèmes paysans, quoique majoritairement représentés, se trouvent être marginalisés dans les discours sur le développement de l'élevage, alors même qu'ils sont source d'innovations, réduisent la vulnérabilité des familles, ou contribuent à des associations durables entre activités agricoles (Herero et al 2007, Steinfeld et al 2010).

Pour sortir de ces discours négatifs vis à vis des «performances» des systèmes d'élevage locaux, il convient alors d'analyser les combinaisons techniques et organisationnelles des systèmes d'élevage tropicaux comme des réponses à des environnements risqués, et en référence à une activité de nature multifonctionnelle, tant à l'échelle de l'exploitation que du territoire (Dedieu et al 2008b, Duteurtre et Faye 2009, Alary et al 2011). De tels indicateurs d'évaluation restent cependant largement à construire dans chaque contexte, pour tenir compte des spécificités locales et des priorités de développement. Les indicateurs zootechniques et économiques classiques doivent ainsi être complétés par des indicateurs environnementaux, culturels et sociaux.

\section{b) Les analyses environnementales}

La recherche a développé un certain nombre de propositions pour l'évaluation des impacts environnementaux des systèmes, qui s'appliquent aux systèmes indépendamment de leur localisation. Dans ce domaine des analyses environnementales normatives, l'Analyse du Cycle de Vie (ISO 14040, ISO 1442) est un bon exemple de méthode qui suscite des attentes importantes pour la caractérisation des systèmes d'élevage et de son utilisation potentielle en certification ou en éco-conception (Mungkung et al 2006). Son approche holistique quantifie les impacts environnementaux d'un produit ou d'un service tout au long de son cycle de vie «du berceau à la tombe» (Guinée et al 2002) en prenant en compte toutes les consommations de ressources et les émissions de polluants. En pratique, un certain nombre de contraintes méthodologiques et la nécessité d'alimenter l'analyse avec des données quantitatives fiables sont des freins à sa généralisation, notamment dans les pays du Sud. Les premiers essais d'application se sont portés sur les filières organisées dans les pays émergents comme sur les filières «porc» et «volaille» au Brésil (Spies 2003). Cette méthode appliquée sur des petites exploitations utilise des indicateurs normatifs (changement climatique, 
acidification, eutrophisation, utilisation d'énergie, écotoxicité...) qui traduisent un effet potentiel sur l'environnement. Elle permet de mettre en évidence des éléments de risque de pollution et met en regard le niveau des impacts avec la productivité des systèmes, la capacité à valoriser les intrants et les logiques d'approvisionnement. De ce fait, elle pourrait permettre l'amélioration des systèmes et proposer des politiques de développement plus adaptées.

Le développement de méthodes d'analyse des empreintes carbone (qui évaluent les émissions de gaz à effet de serre associées aux productions) est patent chez certaines enseignes de la grande distribution (Tesco en Grande Bretagne, ou Casino en France) qui incitent les filières de production animales du Sud (volailles par exemple) à s'interroger sur leur capacité à répondre à des cahiers des charges à venir et à anticiper les demandes de leurs principaux clients (Mungkung et al 2010). Ce type de pratiques de certifications, piloté par l'aval n'est pas sans risques pour les producteurs, comme le montrent des études de vulnérabilité (réalisées en production végétale) qui prennent en compte le transport, l'économie nationale et les spécificités des systèmes de production (Edwards-Jones et al 2008).

c) Evaluation et certification de processus de production

Les systèmes d'élevage en régions chaudes alimentent les flux des grands échanges économiques internationaux. C'est particulièrement le cas pour les productions de masse de pays émergents qui font l'objet de demandes importantes sur des marchés européens et mondiaux, comme la volaille (Brésil, Thaïlande), le porc (Brésil, Vietnam), les crevettes (Thaïlande, Brésil, Vietnam), le Pangasius (Vietnam). Associés à ces échanges, des systèmes de contrôle et de normes ont d'abord été mis en place afin d'évaluer ces élevages sur la qualité de leurs produits et de garantir des niveaux de sécurité et de traçabilité, et ainsi leur permettre d'atteindre les marchés recherchés. Avec l'avènement du développement durable, les systèmes de certification se sont appropriés de nouveaux concepts et ne se contentent plus des pratiques habituelles de traçabilité et d'analyse de points critiques (HACCP), mais se sont ouverts aux impacts environnementaux et sociaux (notamment via les conditions de travail). Ainsi GLOBALGAP, un des leaders de la labellisation business to business en agroalimentaire, propose la certification de producteurs dans les filières de production animales, sur la base de critères de bonnes pratiques agricoles étendues à quelques aspects environnementaux ou sociaux. Ces approches sont basées sur un cahier des charges, sous forme de check-lists d'indicateurs, associé à des systèmes d'audits. Les indicateurs pris en compte portent notamment sur les systèmes de traçabilité au sein de l'élevage mais aussi sur la notion de «rejets, gestion des pollutions, recyclage» ou «santé, sécurité, bien-être des travailleurs». En pratique, même si ces organes de certification s'en défendent, ce type d'approches s'adresse aux industriels de la production animale, à des filières très organisées, voire à des groupements de producteurs, essentiellement dans des systèmes hors-sols ou apparentés (aquaculture), cherchant à s'affranchir au maximum des contraintes et des variations de l'environnement, avec une vision très occidentale de l'élevage. Plusieurs conséquences sont à tirer du développement de ces schémas d'évaluation. Le premier aspect est celui de l'exclusion de fait des petits producteurs incapables de supporter le coût et la lourdeur de ce type de certification. Par ailleurs, ils conduisent à la généralisation de normes basées sur des schémas de qualité propres aux marchés mondiaux qui discréditent des pratiques jugées à risque mais qui ont leur logique dans des contextes locaux. Ces schémas de certification, rarement négociés avec les producteurs, se transforment en des systèmes de contraintes qui, s'ils orientent les systèmes de production animale, peuvent se révéler néfastes à l'échelle des petites structures (coût des intrants, modification des règles sur les marchés locaux...).

\section{3 / Plasticité et développement}

La question de la plasticité dans les processus de conception revêt deux points de débat : le premier concerne la capacité de généralisation de l'innovation au-delà du système d'élevage de référence; le second a trait à la prise en compte des aléas et incertitudes.

Ainsi qu'il a été évoqué plus haut, les critères de diversité des systèmes d'élevage en régions chaudes couvrent des domaines socio-économiques (les fonctions de l'élevage notamment), biotechniques, et spatiaux (la mobilité notamment). On peut alors se poser la question de la façon dont l'activité de conception intègre la diversité. L'amélioration recherchée (cf. la conception réglée) concerne-t-elle tout ou partie de la diversité des systèmes existants? La voie nouvelle explorée (cf. la conception innovante) pourraitelle donner lieu à transition (Lamine et Bellon 2009) pour tout ou partie des systèmes existants? Dans ce dernier cas, le débat porte en effet moins sur la mise au point d'un système nouveau et original que sur les apprentissages (Berkes et Turner 2006) permettant d'engager l'évolution du système vers une nouvelle direction, de nouvelles façons de faire. On sait d'expérience en France qu'un processus de modernisation à modèle unique tel que celui de l'intensification des trente glorieuses contient un fort pouvoir d'exclusion (tous n'y accèdent pas et ne peuvent pas bénéficier des mesures d'accompagnement). Si cette exclusion a pu être absorbée par l'emploi industriel et de services dans notre pays, la situation a de grands risques d'être différente en régions chaudes. Il faut donc pouvoir questionner la conception de systèmes durables relativement à la diversité des systèmes et des modèles de développement (Jollivet 1988).

Le deuxième versant de la plasticité est celui de la prise en compte des aléas et incertitudes dans l'activité de conception. Ces aléas et incertitudes portent la plupart du temps sur :

- le climat (variations climatiques mais également occurrence d'évènements extrêmes comme les cyclones en DOM),

- les prix des produits et des intrants,

- l'occurrence de maladies animales (Ingrand et Faye 2004),

- les politiques agricoles et foncières.

Cette prise en compte des aléas doit retentir sur la production d'indicateurs d'évaluation de la durabilité des systèmes d'élevage : résilience/flexibilité des systèmes d'élevage (Dedieu et al 2008b, Dedieu et Ingrand 2010), stabilité de la production et du revenu (Mosnier et al 2009).

Le développement renvoie selon Béguin (2007) aux interactions entre concepteurs et utilisateurs dans le processus de conception-évaluation. Cette interaction, s'appuyant sur des méthodes participatives, peut être mise en œuvre très en amont dans le prototypage d'un système nouveau (Vereijken 1997) ou la définition de scénarios à explorer (Etienne 2010), notamment en mettant autour de la table des porteurs d'enjeux aux intérêts différents. Elle peut également être d'aval, dans la matérialisation d'un conseil entre producteur et conseiller agricole, le premier ayant à faire valoir ses points de vue et des connaissances dans la formulation d'un conseil adapté (Röling et De Jong 1998). Le développement couvre en général plusieurs phases d'interaction (Etienne 2010) entre les chercheurs (et leurs connaissances scientifiques) et les utilisateurs avec leurs savoir-faire, finalités et représentations. Le débat peut s'appuyer sur plusieurs types de dispositifs de recherche comme le diagnostic 
de situations réelles, le prototypage, l'expérimentation-système (Dedieu et al 2008c, Benoit et Laignel 2009, Coquil et al 2009) ou de modélisation/simulation (par exemple Mar-tin et al 2009). Dans ce cadre, la question de l'évaluation du système joue un rôle central dans les échanges entre parties prenantes du processus de conception. En effet, malgré leur nombre élevé, on peut constater que l'application des ca-dres normatifs d'évaluation des systèmes échoue généralement, dans la mesure où ces cadres ne sont pas complètement aptes à faire évoluer les pratiques dans le sens du développement durable, mais seulement dans le sens de l'amélioration de quelques indicateurs (pour répondre à un cahier des charges par exemple). Ils peuvent conduire à une prise de conscience, mais qui aura du mal à se traduire en réalisations concrètes, s'il n'y a pas une adhésion forte à la démarche d'évaluation. Il s'avère en effet qu'une meilleure compréhension du contexte et un meilleur partage des enjeux ainsi que l'appropriation d'une décision améliorent le processus même de la décision et conduisent à un meilleur engagement des acteurs qui prennent conscience du rôle de chacun et donc d'eux-mêmes. C'est en particulier le cas dans les systèmes agricoles.

En ce qui concerne l'élevage, la prise en compte des logiques d'action des éleveurs, des objectifs multiples et des jeux de contraintes est nécessaire pour une amélioration majeure qui conduit à mieux comprendre les démarches propres de l'éleveur. Les recherches sur l'évaluation des systèmes agricoles se tournent donc vers des approches participatives, où l'acteur participe au processus de recherche, et devient à la fois objet et sujet dans ce processus. Les acteurs ne sont pas seulement les éleveurs, mais aussi les autres personnes concernées par l'activité au travers des bénéfices ou des nuisances qu'ils supportent. De ce fait, apparaissent des schémas de co-construction de systèmes d'évaluation, qui aident les acteurs concernés à définir le cadre le plus pertinent et des indicateurs négociés à partir desquels les systèmes seront évalués. Ces schémas facilitent aussi la prise en main et l'application par les acteurs d'un cadre de développement durable de leur activité (souvent dans leur territoire). Par ailleurs, ils permettent de contourner ou de faire évoluer les systèmes d'évaluation normatifs et les schémas de certification imposés par exemple par les instances internationales (Rey-Valette et al 2008). Des exemples de ce type de démarche seront présentés dans la section 3 .

\section{4 / Conclusion}

La conception de systèmes durables, qu'elle soit réglée ou innovante, doit être explicite sur ce qu'elle intègre de connaissances sur les systèmes réels, leurs caractéristiques et leur diversité. La première partie de cet article a montré combien les systèmes étaient divers et surtout que les fonctions dévolues à l'élevage pouvaient être variables et pour le moins éloignées du cadre dominant d'un élevage à fonction de production. Par ailleurs, la gestion de ces systèmes ne relève pas forcément uniquement d'un chef d'exploitation au style «entrepreneur», (Commandeur et al 2006) qui n'est d'ailleurs qu'une figure parmi d'autres y compris en Europe, En régions chaudes, il existe des farming styles (Commandeur 2006) plus complexes et hétérogènes dont il importe de clarifier les caractéristiques.

Compte tenu des finalités de l'élevage, de ses modalités d'association avec d'autres activités, et de ses modalités d'ancrage territorial, le niveau d'échelle strict du système d'élevage dans l'exploitation, est plutôt abordé dans le cadre de projets finalisés sur des questions concrètes, plutôt que traité de façon générique. Il s'agit là de raisonner le développement durable comme une direction à prendre (Vavra 1996) et d'instrumenter la réflexion relativement à cette direction, plutôt que de produire des indicateurs à valeur universelle, qui gommeraient en partie les effets de localité et de diversité qui marquent les régions chaudes. Dans ce cadre, l'évaluation n'est pas une démarche neutre. La batterie des domaines est large et rarement explorée complètement, mais finalisée par une réflexion sur le développement (dans le sens d'échange et d'apprentissage). Différents niveaux d'échelle (exploitation, territoire, filière, pays et continents) sont à prendre en compte. Ainsi, l'évolution des pratiques d'évaluation tend à impliquer de plus en plus les acteurs du système de production, dans une co-construction de ses modalités, qui permet une appropriation des objectifs de cette évaluation et facilite la mise en place de démarches d'amélioration des systèmes.

\section{3 / Différents exemples de démarches de conception de systèmes durables en régions chaudes}

Nous présentons dans ce texte plusieurs démarches de conception/évaluation de systèmes d'élevage permettant chacune d'éclairer des débats différents :

La première démarche, adoptée aux Antilles (INRA, Unité de Recherches Zootechniques, URZ), est l'illustration d'une démarche de conception «réglée» présentée au travers de deux exemples. Elle se base sur une caractérisation fine des systèmes biotechniques existants à travers la cristallisation de nombreuses connaissances à la fois scientifiques (expérimentations analytiques et/ou expérimentations systèmes) et empiriques (enquêtes de terrains et expertises diverses). Elle vise à améliorer les systèmes existants au regard des contraintes liées à l'exiguïté du territoire en milieu insulaire dans le premier exemple (les études de cas banane et canne à sucre), et l'élaboration de systèmes d'élevages écologiquement intensifs dans le second exemple (systèmes d'élevage caprins au pâturage).

La seconde démarche, à la Réunion (CIRAD, Unité Systèmes d'Elevage méditerranéens et tropicaux, SELMET), montre l'intérêt d'introduire des phases participatives dans l'élaboration du modèle de représentation et d'évaluation du système. Ce modèle traite des améliorations à apporter au fonctionnement de systèmes d'élevage très intensifs, construits autour de logiques de filières, et qui doivent évoluer par une meilleure intégration des enjeux environnementaux, liés aux excès de nutriments et aux questions d'autonomie énergétique. Cette problématique est cruciale dans les milieux insulaires à forte densité agricole et humaine, mais également dans l'ensemble des bassins d'élevage intensif.

La troisième démarche, en Amazonie (CIRAD, Unité Green), consiste en une approche participative de co-construction du modèle d'évaluation. Elle est le fruit d'un partenariat ancien entre la recherche agronomique et les populations agricoles des fronts pionniers. Elle explore les interactions entre fonctionnement des systèmes d'élevage et la dynamique des territoires, avec en trame de fond, la pression internationale forte sur le maintien des surfaces forestières, dans la conception de systèmes d'élevage.

Ces trois démarches permettent ainsi d'illustrer les différents éléments du processus de la conception/évaluation de systèmes durables évoqués (Béguin, ibid.), dans un cadre de conception assistée par la modélisation. Nous y reviendrons dans la discussion.

\section{1 / Conception de systèmes de polyculture-élevage intensifs durables aux Antilles}

En Guadeloupe et en Martinique, les exploitations de type polyculture-élevage représentent environ $80 \%$ des exploitations présentes sur le territoire (Stark et al 2010). Généralement de petite taille, elles ont pour la plupart comme principal objectif d'optimiser les 
Figure 2. Représentation schématique de l'utilisation de la canne à sucre comme base de l'alimentation animale (Adaptée de Gourdine et al 2008).

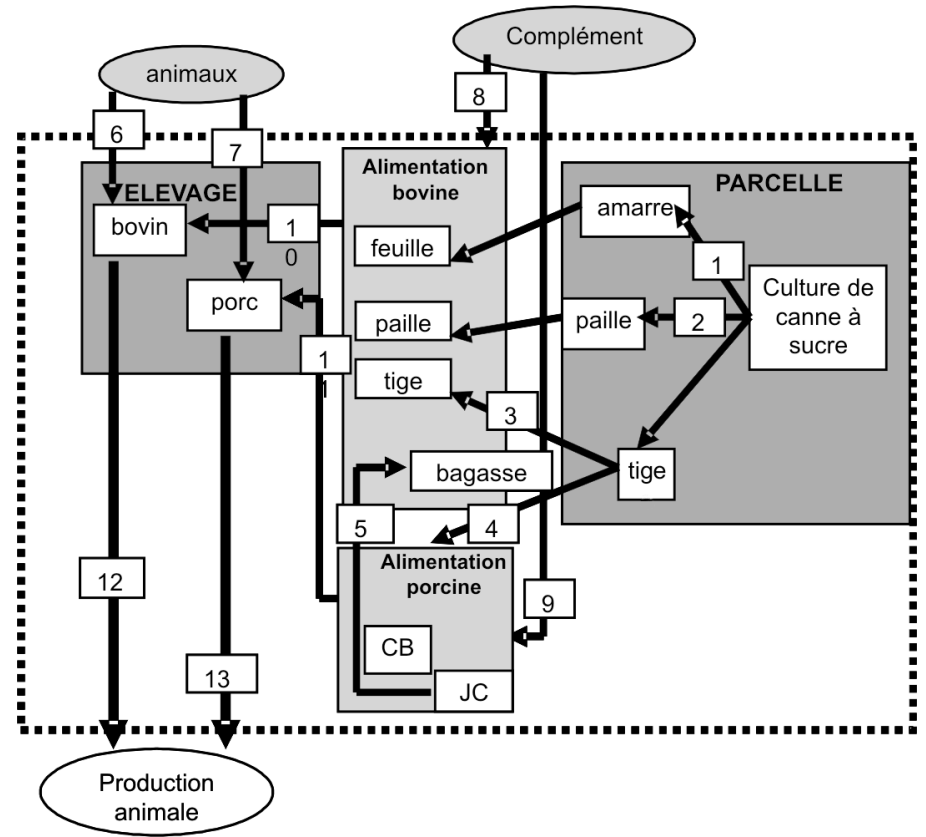

$\mathrm{CB}=$ canne broyée $; \mathrm{JC}=$ Jus de canne $; 1$. Feuilles vertes de canne à sucre disponibles pour l'alimentation bovine ; 2 . Feuilles sèches de canne à sucre disponibles pour l'alimentation bovine ; 3 . Tiges de canne à sucre disponibles pour l'alimentation bovine ; 4 . Tiges de canne à sucre disponibles pour l'alimentation porcine en mode CB ou JC ; 5 . Utilisation de la bagasse, obtenue après pressage lors de la préparation du jus de canne pour les porcs, pour l'alimentation bovine ; 6. Entrée de bovins ; 7. Entrée de porcs ; 8. Entrée de compléments alimentaires (azotés) pour l'alimentation bovine ; 9 . Entrée de compléments alimentaires (azotés) pour l'alimentation porcine ; 10. Utilisation des composantes de la canne à sucre pour l'alimentation bovine ; 11. Utilisation des tiges de canne à sucre sous forme de CB ou de JC pour l'alimentation porcine ; 12. Production de viande bovine ; 13. Production de viande porcine.

productions végétales et animales présentes, en accentuant la complémentarité entre ressources végétales et ateliers d'élevages. Ainsi, les résidus des cultures à destination de l'alimentation humaine, pouvant être complétés par des cultures exclusivement destinées à l'alimentation animale, sont valorisés par des animaux. En retour, le fumier produit sert d'apport en matière organique pour les cultures. Dans une première phase, les recherches menées à l'INRA (URZ) ont porté sur la détermination de la valeur alimentaire des ressources végétales (coproduits de différentes cultures) ainsi que des niveaux de performances des animaux. Ces recherches ont permis de constituer des bases de données conséquentes et de caractériser les relations (flux de matières) existantes entre les compartiments sols, végétaux et animaux des systèmes polyculture-élevage. Des approches par modélisation ont ensuite été développées afin de concevoir et d'évaluer des systèmes représentatifs de ceux présents dans la zone. Deux modèles, centrés sur le système biotechnique ont été construits. Le premier (figure 2) avait pour objectif de représenter une exploitation agricole produisant à la fois des porcs, des ruminants et de la canne à sucre afin d'optimiser l'utilisation des cannes non commercialisées comme ressource principale pour l'alimentation animale (Gourdine et al 2008).
Le second modèle (figure 3 ) visait à étudier, dans un contexte de concurrence mondiale déséquilibrée, les pistes de conversion d'exploitations de type monoculture bananière en exploitations polyculture-élevage valorisant la banane. Une jachère sanitaire était introduite pour casser le cycle des parasites inféodés au bananier. Ces modèles étaient focalisés principalement sur les ateliers de production animale et considéraient les flux de matière et d'énergie générés par l'exploitation depuis la disponibilité de l'aliment jusqu'à la sortie des effluents. Compte tenu de l'approche ex ante retenue, les jeux de données indépendantes permettant une validation complète de ces modèles n'étaient pas toujours disponibles. Ainsi, seules les vitesses de croissance des porcins (modèle canne à sucre) et des caprins (modèle banane) ont fait l'objet d'une validation externe. Cette validation partielle montre que les deux modèles prédisent de manière satisfaisante la production animale. Les simulations montrent qu'un hectare de canne à sucre permettrait de produire respectivement 1,88 et 0,41 Tonnes de poids vif de porcins et de bovins par an. De même, la transformation d'un hectare de banane en système polyculture-élevage permettrait des chargements de $1,12,0,29$ ou 0,42 Tonnes de poids vif à l'hectare, respectivement pour des bovins Créole, des caprins Créole ou des ovins Martinik. Ces deux modèles étant statiques, un effort de recherche est en cours sur l'évolution des ressources végétales afin de les rendre dynamiques et sensibles aux aléas climatiques miques et sensibles aux aléas c

Figure 3. Représentation schématique de l'utilisation des coproduits d'une exploitation bananière comme base de l'alimentation des ruminants (adaptée de Archimède et al 2010).

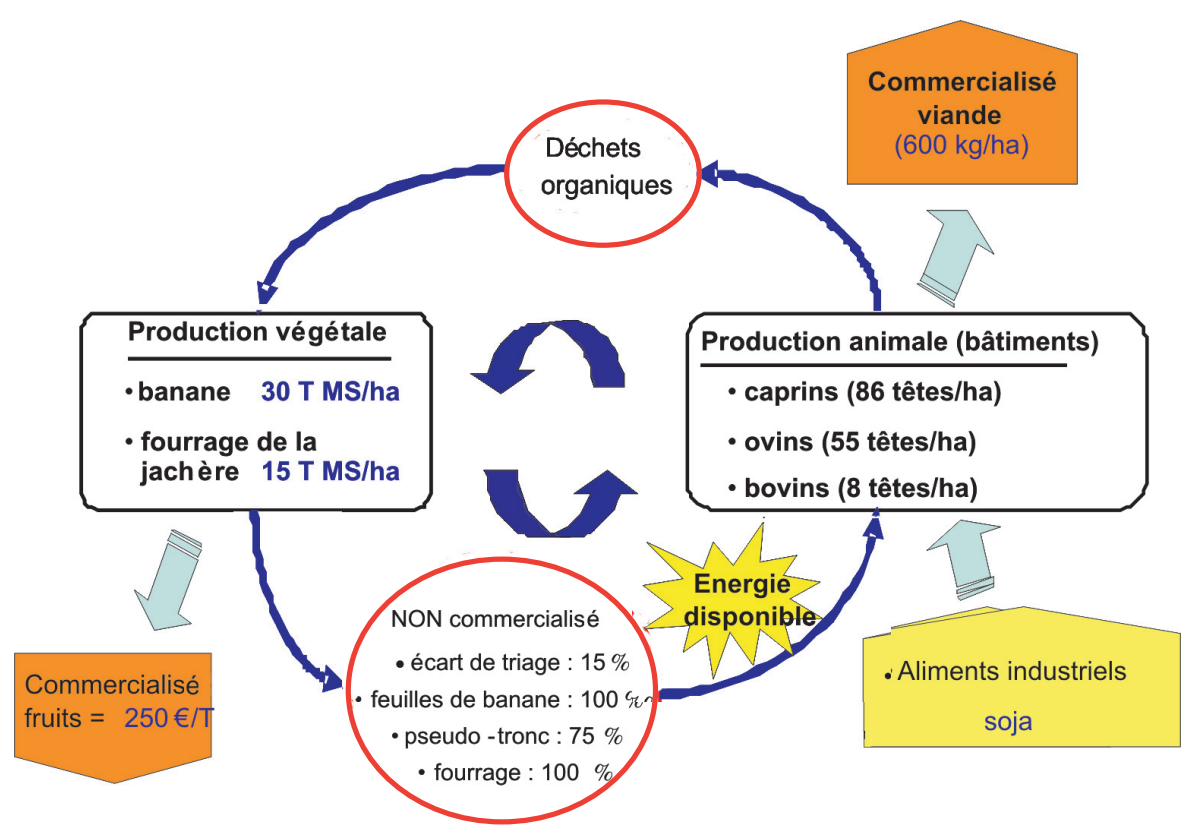


(sécheresse, ouragans...) qui sont relativement fréquents en zone tropicale.

\section{2 / Conception de systèmes d'élevage caprins au pâturage}

La conduite au pâturage est le mode de production animale le plus répandu dans la Caraïbe. Une méthode basée sur l'étude $i$ ) de la prairie (conduite agronomique des parcelles, structure prairiale, ingestion, valeur alimentaire), ii) de la zootechnie des troupeaux (reproduction, alimentation, mode de conduite), et iii) de leurs interactions, a été développée par Alexandre et al (2002). Cette méthode s'inspirait des principaux concepts de l'approche biotechnique des systèmes d'élevage rappelés par Dedieu et al (2008a). Ainsi, des systèmes intensifs d'élevage de caprins Créole au pâturage ont pu être élaborés et testés en unité expérimentale (Mahieu et al 2008). Toutefois, à l'heure actuelle, ces systèmes se heurtent à des impasses quant à leur durabilité. En effet, la gestion du parasitisme, de la pérennité des prairies ou de la variabilité de l'offre fourragère passe encore par un recours important à des intrants généralement importés (anthelminthiques, fertilisants, irrigation, mécanisation...). Certains de ces freins pourraient être levés par des innovations dans la conduite du système, telles que l'association d'espèces ou de stades physiologiques, en cours d'évaluation. Les nombreuses données collectées durant la phase expérimentale, dans divers systèmes de pâturage, mono ou plurispécifiques, plus ou moins intensifs, avec des ovins ou des caprins, ainsi qu'avec des bovins, à divers stades physiologiques, constituent une base informationnelle originale et conséquente (figure 4). Cette base permettra i) d'analyser différents itinéraires techniques de conduite d'ovins et caprins au pâturage, ii) de modéliser les systèmes de pâturage à base de ruminants, et iii) d'élaborer et optimiser de nouveaux modes de gestion des ressources prairiales et des troupeaux permettant une production durable. La modélisation mécaniste du pâturage par les petits ruminants a déjà fait l'objet de travaux. Ainsi, Baumont et al (2004) ont adapté le modèle mécaniste du mouton à l'auge (Sauvant et al 1996) au contexte du pâturage, puis au troupeau pâturant, en milieu tempéré. Ce travail constitue une base au développement d'un modèle spécifiquement adapté à la problématique des systèmes pâturés en milieu tropical humide (contraintes biotiques et abiotiques). Cette adaptation sera innovante par l'intégration dans une même démarche de modélisation, à la fois des dimensions parasitaires et des associations entre espèces de ruminants (Mahieu et Aumont 2009). Le principal enjeu interdisciplinaire est de caractériser et de quantifier les interactions dynamiques existantes entre les différents compartiments des systèmes pâturés, ainsi que leurs facteurs de variations. Cette caractérisation doit permettre d'aboutir à terme à une représentation du système permettant une meilleure compréhension de ses méca- nismes intrinsèques. Elle devrait aussi permettre d'évaluer ex ante différents scénarii en vue d'identifier les freins à la production de caprins au pâturage en zone tropicale humide et par là même, de préciser les besoins spécifiques de recherche.

\section{3 / Co-conception réglée de systèmes laitiers respectueux de l'environnement à la Réunion}

A l'image de ce qui risque de se produire d'ici 40 ans dans de nombreux contextes tropicaux, l'île de la Réunion présente un espace agricole contraint par une densité de population élevée et en plein essor. Pour tenter de répondre au mieux à la demande locale en produits laitiers, l'Elevage Bovin Laitier (EBL) s'est développé par une utilisation importante d'intrants (concentrés, engrais minéraux...). Portées par des enjeux régionaux de conservation des milieux naturels et d'autonomie énergétique, les premières évaluations de l'impact environnemental en terme d'excédents azotés et de consommation d'énergies non renouvelables des systèmes d'exploitation réels, avaient soulevé la question de l'incidence des pratiques d'élevage sur ces critères environnementaux (Vayssières et al 2006, Vigne et al 2009). Un modèle de flux de biomasse, intitulé GAMEDE, a été construit avec pour objectif initial de concevoir des systèmes d'élevage plus respectueux de l'environnement. Il représente, à un pas de temps quotidien, l'ensemble des pratiques de l'éleveur et leurs conséquences

Figure 4. Description sommaire de la base de données URZ sur élevage au pâturage des caprins.

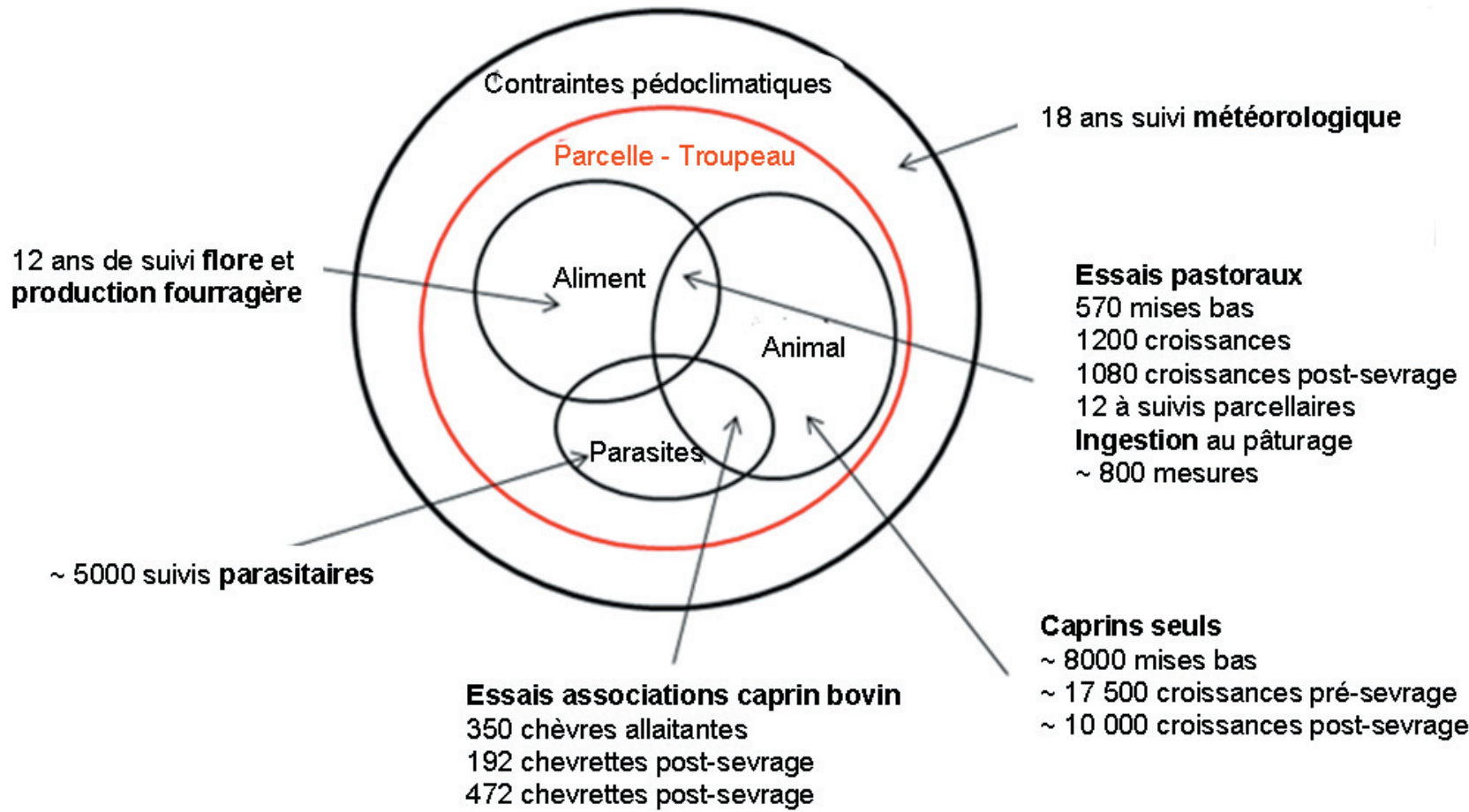


Figure 5. Cycle de conception de systèmes d'élevage basés sur une confrontation entre systèmes réels et modélisés.

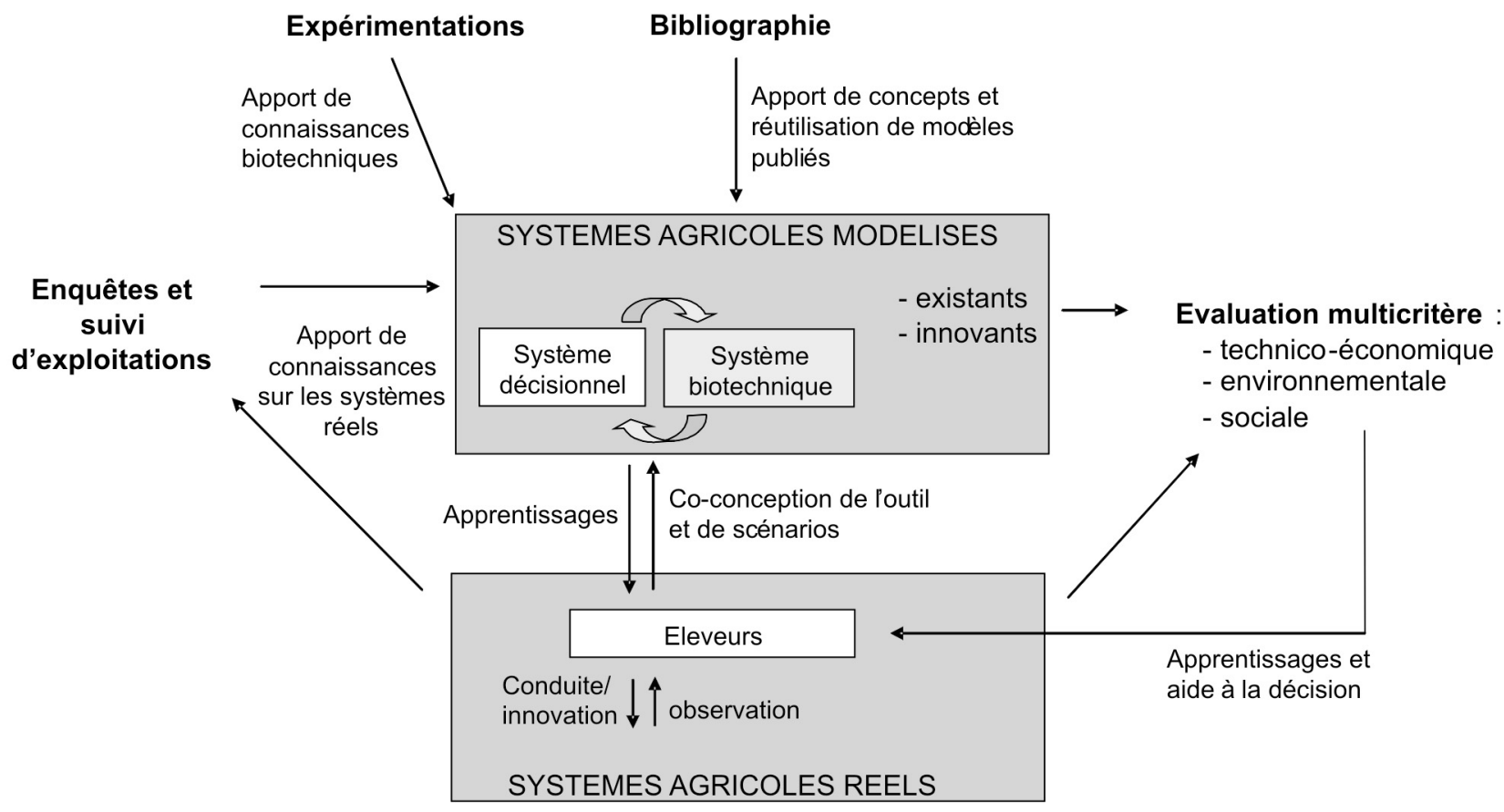

sur les flux dans l'exploitation, et entre l'exploitation et son environnement (Vayssières et al 2009b).

a) Démarche de conception assistée par simulateur

Dans une approche de recherche participative, GAMEDE a été le support de conception de systèmes d'élevage innovants. La démarche de conception a fait l'objet d'un ensemble de cycles d'échanges de connaissances impliquant des éleveurs, des techniciens et des chercheurs. Cette démarche de conception (schématisée en figure 5) est basée sur $i$ ) une connaissance profonde des systèmes réels, ii) la co-construction d'un modèle de représentation fine de la gestion des systèmes de production, iii) la mise en débat d'indicateurs multiples d'évaluation des systèmes observés et simulés, iv) la simulation interactive d'une gamme large de systèmes réels et innovants dans une perspective d'apprentissage et d'aide à la décision.

Deux types d'enquêtes en exploitations ont été réalisés pour connaître de façon approfondie les systèmes réels : des immersions et des suivis d'exploitations. Les immersions (une semaine par EBL en année 1), inspirées par les approches ethnographiques, ont été conçues pour identifier les stratégies, les plans d'action et les règles de décision quotidiennes des éleveurs (Vayssières 2004). Les suivis d'élevage (un jour par EBL tous les deux mois pendant 2,5 ans en années 1 à 3) ont permis i) l'analyse des pratiques et plus particulièrement la confrontation de la conduite planifiée à la conduite réalisée pour mettre en évidence des règles d'ajustement, ii) l'observation de variables d'état du système de production telles que les stocks de fourrage et la composition du troupeau. Ces deux types d'enquêtes ont concerné 6 éleveurs choisis pour représenter la diversité des systèmes d'élevage en référence à un zonage à dire d'experts et une typologie de pratiques (Vayssières et al 2010).

Ces 6 éleveurs ont également étroitement participé à la conception de GAMEDE dans le cadre de réunions collectives $(1 / 2$ journée tous les 3 mois pendant 2,5 ans en années 2 à 4). La connaissance apportée par les éleveurs lors des enquêtes sur leurs systèmes réels a été traduite en règles de décision et en processus biotechniques à prendre en compte dans le modèle. Les réunions collectives étaient basées sur une restitution progressive des avancées du travail de modélisation associant la conception et les simulations. Chacune des étapes de modélisation a été discutée et validée par le groupe (Kerdoncuff 2007, Vayssières et Lecomte 2007). Lors de ces réunions, les indicateurs calculés par le modèle ont été vivement débattus. Alors que les chercheurs avaient initialement prévus de privilégier les critères environnementaux en référence à des approches de type «bilan de nutriments aux portes de la ferme» et «Analyse de Cycle de Vie», les éleveurs ont souhaité compléter ces indicateurs par des indicateurs technico-économiques et sociaux plus proches de leurs systèmes de référence. Les indicateurs technico-économiques demandés correspondent aux indicateurs classique- ment restitués par leur encadrement technique tels que la productivité des prairies, la productivité laitière et la marge brute. Les indicateurs sociaux correspondent à la charge de travail générée par le système de production et la part du travail d'astreinte. Ainsi, partant d'une approche très environnementaliste, les éleveurs ont conduit les chercheurs vers une évaluation des trois piliers de la durabilité.

b) Connaissances générées en cours de conception

Ce projet de co-conception a également fait l'objet d'apprentissages qui diffèrent selon les acteurs impliqués. Alors que la majorité des solutions jusque là imaginées par la recherche à la Réunion étaient technologiques ou se situaient à un niveau stratégique, ces travaux ont montré qu'il est possible de réduire l'impact environnemental des systèmes d'élevage par un simple changement des pratiques de conduite (innovation organisationnelle). Or, ce changement des règles de décision opérationnelles est plus facilement adopté par des éleveurs qu'un bouleversement nécessairement plus profond du système de production induit par un changement de stratégie et/ou de structure d'exploitation. La place déterminante des dimensions sociales pour la compréhension des systèmes existants et pour le conseil en élevage a été retenue par les techniciens d'élevage comme étant un point essentiel révélé par ce projet de conception. En effet, l'adoption de systèmes d'élevage plus respectueux de l'environnement 
(Vayssières et al 2009a) et plus largement la transmission des exploitations sont fortement déterminées par des questions de charge de travail (Seegers et al 2006).

Quant aux éleveurs, au-delà de leurs objectifs personnels de viabilité économique et de vivabilité sociale, ils se sont appropriés les enjeux environnementaux régionaux initialement portés par les chercheurs. Ces enjeux se retrouvent dans les systèmes aujourd'hui mis en œuvre par les éleveurs du projet, qu'il s'agisse de systèmes conçus autour du modèle GAMEDE ou de systèmes plus innovants imaginés pas les éleveurs et non simulables (car basés sur la pluriactivité agricole ou non agricole). Ces apprentissages déterminent, déjà aujourd'hui (trois ans après le projet), via les trois types d'acteurs impliqués, la nature et la durabilité des systèmes laitiers innovants.

\section{4 / Modélisation et approches participatives pour la conception de systèmes d'exploitation assu- rant la pérennité de la zone forestière en Amazonie}

Une tendance forte de la recherche en Amazonie est la construction de modèles en vue de comprendre les processus de déforestation. A partir de données macroéconomiques et d'analyses de télédétection, les chercheurs quantifient ainsi les corrélations entre des variables (Kaimowitz et Angelsen 1998) et participent au débat international sur les moyens de préserver la forêt amazonienne
(Poccard-Chappuis et al 2005). Par exemple, Nepstad et al (2002) projettent les déforestations futures en fonction de probabilités de création de nouvelles routes ou l'asphaltage des pistes. Ces modèles n'analysent pas les mécanismes fondamentaux entraînant la déforestation. Enfin, les acteurs ne sont pas représentés à ces échelles, ou au mieux sous forme de densité et de flux de population. De fait, les connaissances des déterminants de la déforestation et des processus décisionnels des acteurs restent rudimentaires (Piketty 2003).

Ferreira (2001) et Ludovino (2002) ont montré grâce à leurs modèles conceptuels que les échecs de colonisation sont plus souvent sociaux que techniques ou économiques. A partir de là, plusieurs approches sociales et participatives voient le jour et mettent en évidence la différence de perception de l'écosystème forestier de la société et de la communauté scientifique. En modélisant le processus décisionnel et les pratiques d'utilisation des terres, Bonaudo (2005) a initié l'usage des Systèmes Multi-Agents (SMA) sur ces problématiques. Depuis, ils fleurissent entre les mains de diverses équipes. Ils sont perçus comme des outils en appui aux approches participatives pour partager les connaissances, tant entre acteurs locaux qu'entre recherche et société. Ils s'appuient sur les stratégies et les décisions des acteurs, à partir d'ateliers participatifs ou d'entrevues. Réalistes et parlants, ils permettent de mieux saisir les activités des acteurs pour explorer des scénarios prospectifs.
Le SMA TransAmazon (Bonaudo et al 2005, Bommel et al 2010) se focalise sur les décisions individuelles des agents pilotes de leur système agricole. Pour modéliser le fonctionnement d'un tel système, les auteurs identifient les objectifs des acteurs ainsi que les éléments déterminants des choix de production. Pour cela, ils identifient les pratiques qui forment la partie apparente de la décision (Yung et Zaslavsky 1992). Pratiques et changements de pratiques constituent des points de repère permettant de reconstituer les stratégies. Ce système décisionnel est simplifié et implémenté dans le SMA, qui propose deux modes de pilotage annuel privilégiant soit les cultures pérennes, soit l'activité d'élevage (Stratégies Planteur ou Eleveur) (figure 6). Chaque mode de pilotage regroupe et ordonne des actions pour implanter des cultures, entretenir le troupeau, conserver une réserve forestière ou l'exploiter (coupe de bois), etc. L'agent choisit son pilotage en fonction d'informations et de contraintes comme la quantité de maind'œuvre familiale (Hostiou et Dedieu 2009), le prix de vente des productions, la disponibilité en terres et le bénéfice prévisionnel. Grâce aux actions des agents et aux changements de pilotage, on retrouve par la simulation des structures d'exploitation semblables à la typologie observée. Outre une représentation spatiale dynamique, elle permet de suivre l'évolution de nombreux indicateurs tant individuels (couverture d'un lot, capital d'un agent, ses ventes...) que globaux (nombre d'exclus, économie de la communauté, surface en

Figure 6. Diagramme d'un système d'exploitation d'élevage amazonien (priorités des activités : planteur ; éleveur ; sans terre) (Bommel et al 2010).

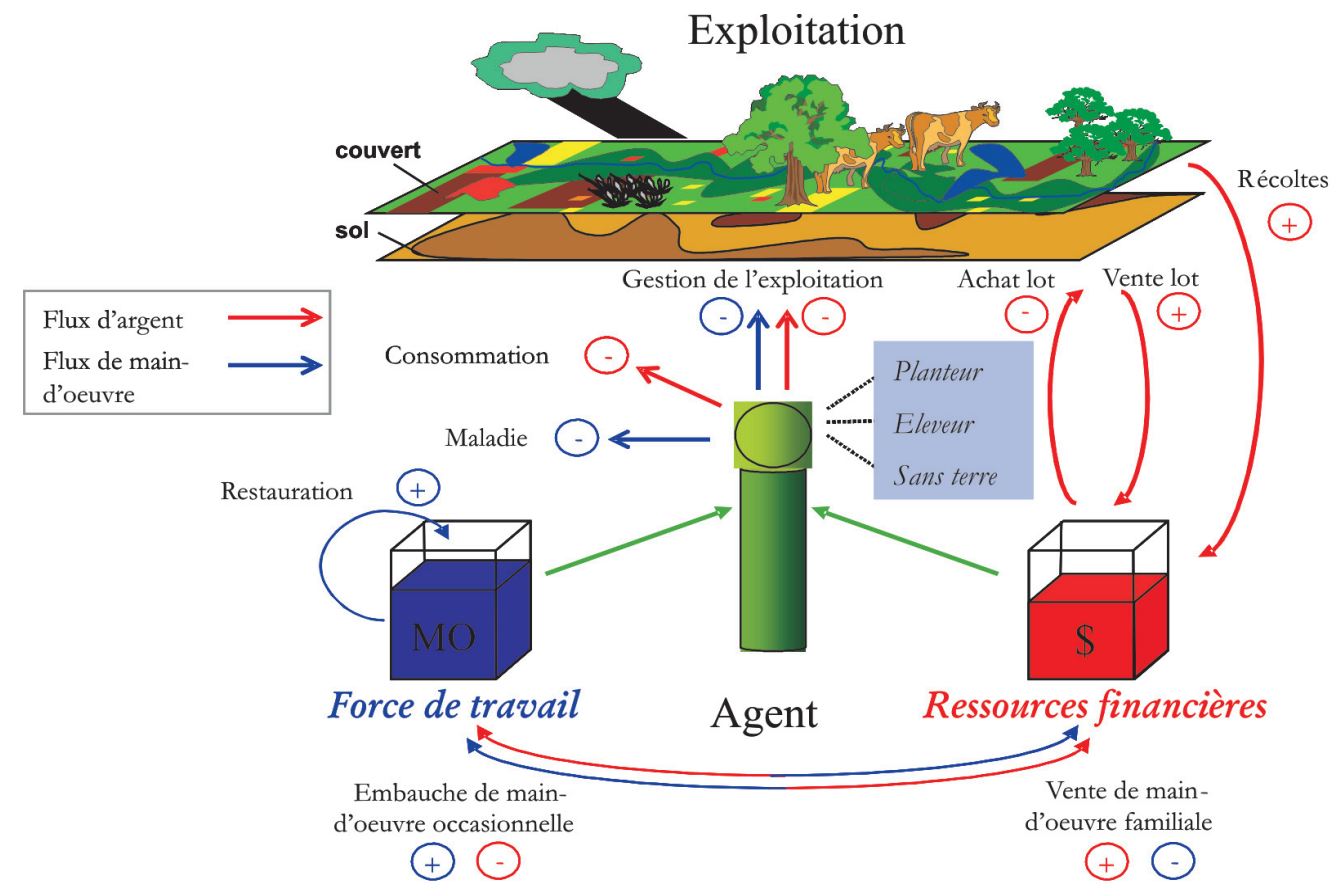


forêt). Les résultats des simulations montrent que, malgré sa faible rentabilité, l'élevage domine. Car, contrairement aux cultures comme le cacao, le troupeau constitue une épargne rapidement mobilisable. L'élevage constitue ainsi une garantie face aux risques économiques ou de santé. Ces phénomènes, non formalisés dans le modèle, émergent des simulations qui montrent par moment des ventes de bétail lors de difficultés familiales.

En se focalisant sur les acteurs, le SMA TransAmazon apporte d'autres points de vue sur les dynamiques des fronts pionniers que les modèles macroéconomiques de corrélation évoqués précédemment (Nepstad et al 2002). Les facteurs (routes, mode de fairevaloir...) mobilisés par ces modèles standards doivent être révisés afin de mieux prendre en compte les raisons qui guident les pratiques de déforestation. On s'aperçoit alors que la gestion du risque est un facteur prépondérant dans le choix des activités agricoles, l'élevage jouant un rôle de sécurité pour la famille. Or, c'est principalement l'élevage extensif qui entraîne l'avancée du front pionnier.

Couplés à des approches participatives, les SMA s'avèrent être des outils pertinents pour révéler et hiérarchiser les facteurs décisifs de la déforestation. Sans se polariser exclusivement à quantifier la déforestation, ces modèles obligent à mieux appréhender le contexte et à imaginer des alternatives.

\section{4 / Discussion - Conclusion}

Les illustrations présentées ne couvrent pas l'ensemble des domaines d'activités de conception/évaluation de systèmes d'élevage durables des équipes INRA et CIRAD citées ! Elles visent surtout à souligner tant la diversité des enjeux de durabilité en milieu tropical que des questions à traiter dans la mise en œuvre de démarches de conception de systèmes.

\section{1 / Cristallisation, plasticité et développement}

Ce qui est cristallisé dans l'activité de conception de systèmes d'élevage durables peut être un approfondissement du domaine biotechnique (exemple de Guadeloupe), une compréhension fine du fonctionnement des systèmes d'élevage dans leurs dimensions humaines et techniques (exemple de la Réunion), ou la mise en relation des stratégies d'éleveurs et des pratiques techniques (ici d'utilisation de l'espace dans le cas de l'Amazonie). Le cas des Antilles est intéressant dans la mesure où l'équipe de recherche vise l'amélioration de deux modèles d'élevage bien différents :

- l'un intensif et spécialisé (l'élevage caprin) dont il s'agit de corriger les défauts vis-à-vis des questions environnementales,

- l'autre qui vise à remettre en débat, sous l'angle de la durabilité, des systèmes traditionnels de polyculture-élevage dans lesquels l'élevage avait comme fonction essentielle la valorisation de résidus et de sous-produits.

La question de la diversité des systèmes que nous avons évoquée dans la première partie, est ainsi prise en compte à travers deux projets de modélisation disjoints. Derrière la notion de plasticité des démarches, il s'agit bien d'expliciter les cibles potentielles et celles qui sont exclues des retombées opérationnelles des modélisations proposées Notamment, les réflexions sont-elles compatibles avec les différentes formes que prend la multifonctionnalité locale de l'élevage ? S'adressent-elles à des formes spécifiques orientées vers la production ou peuvent-elles aider à faire évoluer des systèmes pour lesquels d'autres fonctions (sécurisation des familles) sont importantes pour la durabilité ? On conçoit ici que la question de la formalisation des finalités et des logiques d'action des éleveurs est une question très sensible pour la recherche : elle implique une pluridisciplinarité réelle et des méthodes adaptées. La méthodologie d'immersion, de suivis réguliers individuels et de rendezvous collectifs, pratiquée dans la cadre de l'étude réalisée à la Réunion apparaît alors essentielle pour formaliser les règles de décision et discuter d'indicateurs d'évaluation partagés entre chercheurs et agriculteurs.

Ce type de méthodologie permet aussi de mettre en évidence le poids du facteur «travail» dans le fonctionnement, l'amélioration des situations ou la mise en œuvre de transitions vers des systèmes plus durables (Dedieu et Serviere 2011). Dans des régions où le travail manuel demeure très présent, la capacité à investir dans du matériel (pot trayeur par exemple), les opportunités de délégation ou de partage du travail sont déterminants de la possibilité de faire évoluer des conduites d'élevage (Cournut et al 2010, Hostiou et al 2010). TransAmazon et GAMEDE proposent quelques éléments de caractérisation du travail. Mais globalement le couplage entre le fonctionnement du système technique et du système travail et le poids des différents contextes et pressions de travail (liés à la dimension de l'activité agricole, la composition des familles, à la pluriactivité ) sont très insuffisamment informés alors qu'ils participent au pilier social de la durabilité et que le travail est reconnu comme un frein majeur à l'innovation (Mak 2001). Les plates-formes de modélisation comme DIESE (Martin Clouaire et Rellier 2009) qui représentent le système décisionnel comme le choix et l'ajustement d'un système d'activités de travail ouvrent, de ce point de vue, des pistes intéressantes pour ne pas laisser dans l'ombre le travail, son organisation et ses liens avec le domaine biotechnique.

La façon dont les éleveurs eux même sont partie prenante du développement de l'activité de conception est assez diverse selon les démarches. En Guadeloupe, la connaissance de la diversité de fonctionnement des systèmes d'élevage apparaît comme un préalable au formatage des objectifs des modèles et des expérimentations biotechniques. Dans le cas des modèles d'Amazonie ou de la Réunion, le développement du modèle lui-même a donné lieu à une participation des éleveurs. C'est cette participation qui a amené les modélisateurs à intégrer les cultures vivrières et la main-d'œuvre dans la formalisation d'un système traitant d'élevage et d'espaces en Amazonie, ou d'introduire les objectifs sociaux et économiques et des indicateurs correspondants dans une modélisation à vocation environnementale à la Réunion. Le fait que les éleveurs aient été progressivement familiarisés avec le modèle GAMEDE et que ce dernier simule de façon réaliste leurs propres systèmes de production (grâce entre autres à une simulation fine des pratiques) a, dans ce dernier cas, facilité la conduite de simulations interactives.

\section{2 / La durabilité : une ques- tion multi-échelle et multi- acteur}

L'exemple de la démarche adoptée à la Réunion montre bien que la question des indicateurs d'évaluation des systèmes demeure complexe, mais ne saurait être abordée sans les acteurs euxmêmes. A l'échelle de l'exploitation, c'est sans doute une voie pour formaliser ce que les éleveurs attendent du fonctionnement de leur système. La démarche permet de préciser quels indicateurs pourraient être pertinents notamment dans le domaine social, lequel ne se prête pas à des propositions normatives extérieures. Les exemples développés ici accordent peu de poids aux réflexions planétaires sur la diminution des gaz à effet de serre ou des enjeux de sécurité alimentaire mondiale. Elles sont surtout justifiées par des problématiques régionales ou locales de développement durable 
posées autour d'une double question de respect de l'environnement et de pérennisation des formes d'élevage qui jouent un rôle dans le développement de sociétés rurales ou périurbaines en occupant l'espace, en maintenant des populations en place. Ces niveaux de réflexion sur la durabilité et le développement de l'élevage doivent être mieux reliés entre eux dans la réflexion sur l'évaluation, sur sa composante experte et normative et sa composante débattue entre acteurs concernés des territoires.

Au final, la conception/évaluation de systèmes d'élevage tropicaux durables est, comme en milieu tempéré, un formidable challenge d'intégration des connaissances tant dans le domaine biotechnique que dans le domaine décisionnel et du travail. Elle interroge sur ce que les équipes peuvent cristalliser et développer compte tenu de leurs cultures de recherches, leurs partenariats pluridisciplinaires et la façon dont elles travaillent avec les acteurs-utilisateurs. Les systèmes tropicaux ont de ce point de vue, des spécificités fortes à faire valoir : dans les fonctionnements biotechniques (ressources et animaux), dans la variété des fonctions de l'élevage pour les exploitants, et dans l'expression même des enjeux et des critères de durabilité. La «résistance paysanne» à des propositions innovantes, orientée vers la production est bien moins de l'immobilisme qu'une traduction de logiques d'élevage où le troupeau remplit des fonctions non productives (autoconsommation, solidarité, épargne) dans un milieu très aléatoire dont il faut pouvoir tenir compte. Si l'intensification écologique (Griffon 2006) est bien un challenge technique audacieux pour relever le défi du changement glo- bal, cette ligne d'innovation devra tenir compte des fonctions multiples de l'élevage, de la nécessité pour les éleveurs de faire avec les aléas et l'incertitude (Lemery et al 2005) des régions chaudes. Ces deux caractéristiques importantes, auquel il faut rajouter la prépondérance d'un travail souvent manuel, amènent à penser l'élevage en référence aux familles et à leurs combinaisons d'activités (lesquelles concourent ensemble à la sécurisation) comme le proposent Herrero et al (2007). A la lumière de ces exemples, les questions d'association élevage-autres activités apparaissent centrales, qu'il s'agisse de la valorisation par l'élevage de toutes les ressources, des coproduits existants (comme en Guadeloupe), de l'intégration de cultures et d'élevage (Guadeloupe, Amazonie), ou enfin de la pluriactivité et de ses implications.

\section{Références}

Alary V., Chalimbaud J., Faye B., 2007. Multiple determinants of milk production in Africa: the example of the diversity of dairy farming systems in the Mbarara area (Uganda). Afr. Dev., 32, 156-180.

Alary V., Duteurtre G., Faye B., 2011. Elevages et sociétés : les rôles multiples de l'élevage dans les pays tropicaux. In : Numéro spécial, Elevage en régions chaudes. Coulon J.B., Lecomte P., Boval M., Perez J.M. (Eds). INRA Prod. Anim., 24, 145-156.

Alexandre G., Archimède H., Boval M., Mahieu M., Aumont G., Xandé A., 2002. A holistic approach to the multi-functionality of the grazing systems in the Carribean. $19^{\text {th }}$ General Meet. Eur. Grassland Fed., La Rochelle, France, 1004-1005.

Alexandre G., Asselin de Beauville S., Shitalou E., Zebus M.F., 2008. An overview of the goat meat sector in Guadeloupe: conditions of production, consumer preferences, cultural functions and economic implications. Livest. Res. Rural Dev., 20, www.lrrd.org/lrrd20/1/ cont2001.htm

Ancey V., Ickowicz A., Manoli C., Magnani S., 2007. Liens entre troupeaux et familles chez les Peuls du Ferlo : indicateurs socioéconomiques des mutations de l'élevage pastoral. Renc. Rech. Rum., 185-188.

Ancey V., Ickowicz A., Toure I., Wane A., Diop A.T., 2009. La vulnérabilité pastorale au Sahel : portée et limite des systèmes d'alerte basés sur des indicateurs. In : L'élevage, richesse des pauvres. Duteurtre G., Faye B. (Eds). Editions Quae, Versailles, France, 117-132.

Archimède H., González-García E. Fanchone A., Tournebize R., Gourdine J.L., 2011. Integrating banana and ruminant production enhance diversification at farm level while diminishing environmental risks of a monoculture model. Agric. Syst., soumis.

Archimède H., Bastianelli D., Boval M., Tran G., Sauvant D., 2011. Ressources tropicales : disponibilité et valeur alimentaire. In : Numéro spécial, Elevage en régions chaudes.
Coulon J.B., Lecomte P., Boval M., Perez J.M. (Eds). INRA Prod. Anim., 24, 23-40.

Bah A. Toure I., Fourage C., Gaye D.I., Leclerc G., Soumare M.A., Ickowicz A., Diop A.T., 2010. Un modèle multi-agents pour étudier les politiques d'affectation des terres et leurs impacts sur les dynamiques pastorales et territoriales au Ferlo (Sénégal). Cah. Agric., 19, 118-126.

Baumont R., Cohen-Salmon D., Prache S., Sauvant D., 2004. A mechanistic model of inta$\mathrm{ke}$ and grazing behaviour in sheep integrating sward architecture and animal decisions. Anim. Feed Sci. Technol., 112, 5-28.

Béguin P., 2007. Innovation et cadre sociocognitif des interactions concepteurs-opérateurs : une approche développementale. Le Travail Humain, 70, 369-390.

Benoit M., Laignel G., 2009. Performances techniques et économiques en élevage biologique d'ovins viande : observations en réseaux d'élevage et fermes expérimentales. In : Numéro spécial, Elevage Bio. Perez J.M. (Ed). INRA Prod. Anim., 22, 197-206.

Berkes F., Turner N.J., 2006. Knowledge, learning and the evolution of conservation practice for social-ecological system resilience. Human Ecol., 34, 479-493.

Blanc-Parmard C., Boutrais J., 1994. A la croisée des parcours : Pasteurs, éleveurs, cultivateurs, Collection Colloques et séminaires, Editions Orstom, Paris, France, 336p.

Bonaudo T., 2005. La gestion environnementale sur un front pionnier amazonien. Thèse de doctorat en zootechnie, INA Paris Grignon, Paris, France, 354p.

Bonaudo T., Bommel P., Tourrand J.F., 2005 Modélisation des fronts pionniers de la Transamazonienne, SMAGET, Bourg-SaintMaurice, France, 21p.

Bonfiglioli A.M., 1984. Dudal, histoire de famille, histoire de troupeau. C.U.P., MSH., 293p.

Bommel P., Bonaudo T., Barbosa T., Da Viega J., Vieira Pak M.P., Tourrand J.F., 2010.
La relation complexe entre l'élevage et la forêt en Amazonie. Une approche par la modélisation multi-agent. Cah. Agric., 19, 104-111.

Commandeur M., 2006. Diversity of swine farming styles: understanding how it is structured. Wageningen Journal of Life Sciences, NJAS, 54, 111-127.

Commandeur M., Le Guen R., Dourmad J.Y., Casabianca F., 2006. La diversité des styles d'élevage porcin : une approche dans les Côtes d'Armor. Journ. Rech. Porcine Fr., 247254.

Coquil X., Blouet A., Fiorelli J.L., Bazard C., Trommenschlager J.M., 2009. Conception de systèmes laitiers en agriculture biologique : une entrée agronomique. In : Numéro spécial, Elevage Bio. Perez J.M. (Ed). INRA Prod. Anim., 22, 221-234.

Coulon J.B., Lecomte P., 2009. Dispositif de recherches INRA-CIRAD sur les productions animales en régions chaudes : contexte, état des lieux et perspectives. in : Enjeux et priorités de la recherche pour l'élevage dans les pays du Sud. Session satellite, Renc. Rech. Rum., 43-50.

Cournut S., Rawski C., Madelrieux S., 2010, Transformation des systèmes d'élevage et du travail des éleveurs, Numéro thématique, Cah. Agric., 19, 309-390.

Dedieu B., Ingrand S., 2010. Incertitude et adaptation : cadres théoriques et application à l'analyse de la dynamique des systèmes d'élevage. In : Robustesse, rusticité, flexibilité, plasticité, résilience... les nouveaux critères de qualité des animaux et des systèmes d'élevage. Sauvant D., Perez J.M. (Eds). Dossier, INRA Prod. Anim., 23, 81-90.

Dedieu B., Servière G., 2011. Les modèles du travail en élevage : points de vue de zootechniciens. In : Le travail en agriculture : son organisation et ses valeurs face à l'innovation. Beguin P., Dedieu B., Sabourin E. (Eds). L'Harmattan, 155-170. 
Dedieu B., Faverdin P., Dourmad J.Y., Gibon A., 2008a. Système d'élevage, un concept pour raisonner les transformations de l'élevage. In : Numéro spécial Anniversaire, 20 ans de recherches en productions animales à l'INRA. Charley B., Herpin P., Perez J.M. (Eds). INRA Prod. Anim., 21, 45-57.

Dedieu B., Chia E., Leclerc B., Moulin C.H., Tichit M., 2008b. L'élevage en mouvement : flexibilité et adaptation des exploitations d'herbivores. Editions Quae, Collection Sciences et Technologie Update, Versailles, France, 294p.

Dedieu B., Louault F., Tournadre H., Benoit M., 2008. Réponse des systèmes d'élevage innovants à la variabilité climatique : une expérimentation en production extensive ovin viande intégrant des préoccupations environnementales. In : L'élevage en mouvement : flexibilité et adaptation des exploitations d'herbivores. Dedieu B., Chia E., Leclerc B., Moulin C.H., Tichit M. (Eds). Editions Quae, Collection Sciences et Technologie Update, Versailles, France, 161-178.

Diman J.L., Naves M., Alexandre G, Zebus M.F., 2006. The diversity of ruminant rearing systems in Guadeloupe: positions within the industry and its sanitary regulations. In: Livestock farming systems: product quality based on local resources leading to improved sustainability. Rubino R., Sepe L., Dimitriadou A., Gibon A. (Eds). Wageningen Academic Publishers, The Netherlands, 355-360.

Diop A.T., Sy O., Ickowicz A., Toure I., 2003. Politique d'hydraulique et gestion de l'espace et des ressources dans la région sylvopastorale du Sénégal (Ferlo). Organisation spatiale et gestion des ressources et des territoires ruraux. CD Rom, Actes du colloque Umr SAGERT, 25-27 février, Montpellier, France, 9p.

Duteurtre G., Faye B., 2009. L'élevage, richesse des pauvres : Stratégies d'éleveurs et organisations sociales face aux risques dans les pays du Sud. Editions Quae, Versailles, France, $286 \mathrm{p}$

Edwards-Jones G., Plassmann K., York E.H., Hounsome B., Jones D.L., Milà i Canals L., 2008. Vulnerability of exporting nations to the development of a carbon label in the United Kingdom. Env. Sci. Policy, 12, 479-490.

Etienne M., 2010. La modélisation d'accompagnement : Une démarche participative en appui au développement durable. Editions Quae, Collection Sciences et Technologie Update, Versailles, France, 384p.

Faye B., Bengoumi M., Barkat A., 2003. Le développement des systèmes camélins laitiers périurbains en Afrique. Atelier Int. sur le lait de chamelle en Afrique. FAO-CIRAD-KARKARA, Niamey, Niger, 5-8/11/03, 115-125.

Ferreira L.A., 2001. Le rôle de l'élevage bovin dans la viabilité agro-écologique et socio-économique des systèmes de production agricoles familiaux en Amazonie brésilienne le cas d'Uruará (Pará, Brésil). Thèse INA Paris Grignon, Paris, France, 188p.

Gibon A., Ickowicz A., 2010. Transformation des systèmes d'élevage extensifs dans les territoires ruraux. Numéro thématique, $\mathrm{Cah}$ Agric., 19, 77-172.

Gibon A., Rubino R., Sibbald A.R., Sorensen J.T, Flamant J.C., Lhoste P., Revilla R., 1999. Livestock farming systems research in Europe an dits potential contribution for managing towards sustainability in livestock farming. Livest. Prod. Sci., 96, 11-31.
Gourdine J.L., Xandé X., Renaudeau D., Gonzalez E., Bructer M., Archimède H., 2008 Exemple d'un fonctionnement biotechnique d'un système culture élevage à base de canne. 4ème Renc. Int. Francophone de l'Association Française de la Canne à Sucre, Guadeloupe (FWI). 10p. http://www.afcas.info/documents T1-a-14_GOURDINE.pdf.

Griffon M., 2006. Nourrir la planète. Pour une révolution doublement verte. Odile Jacob Ed., Paris, France, 456p.

Grimaud P., Mpairwe, Chalimbaud J., Messad S., Faye B., 2007. The place of Sanga cattle in dairy production in Uganda. Trop. Anim. Health. Prod., 39, 217-227.

Guerin H., Faye B., 1999. Spécificité de la problématique périurbaine pour les systèmes d'élevage. In : Agriculture périurbaine en Afrique subsaharienne. Moustier P., Mbaye A. De Bon H. Guerin H., Pagès J. (Eds). Actes de l'atelier CIRAD-CORAF, 20-24 avril 1998, Montpellier, France, 43-49.

Guinée J.B., Gorrée M., Heijungs R., Huppes G., Kleijn R., de Koning A., van Oers L., Wegener Sleeswijk A., Suh S., Udo de Haes H.A., de Bruijn H., van Duin R., Huijbregts M.A.J., 2002. Handbook on life cycle assessment. An operational guide to the ISO standards. Dordrecht. The Netherlands: Kluwer Academic Publishers, 704p.

Herrero M., Gonzalez-Estrada E., Thornton P.K., Quiros C., Waithaka M.M., Ruiz R. Hoogenboom G., 2007. IMPACT: Generic household-level databases and diagnostics tools for integrated crop-livestock systems analysis. Agric. Syst., 92, 240-265.

Hostiou N., Dedieu B., 2009. Diversity of forage system work and adoption of intensive techniques in dairy cattle farms of Amazonia. Agron. Sustain. Dev., 29, 535-544.

Hostiou N., Dedieu B., Madelrieux S., Pham K.D., Vu Trong B., 2010. Relations entre organisation du travail et taille des exploitations laitières : une étude à Moc Chau (Vietnam). Cah. Agric., 19, 323-333.

Ickowicz A., Bah A., Bommel P., Etienne M. Gibon A., Laseur J., Morales H., Touré I. Tourrand J.F., 2010. Facteurs de transformation des systèmes d'élevage extensif des territoires étude comparée des dynamiques locales sur trois continents. Cah. Agric., 19, 127-134.

INRA-CIRAD, 2009: Agrimonde: Agricultures et alimentations du monde en 2050 scénarios et défis pour un développement durable. 2 ème édition, décembre 2009, Note de synthèse, Institut National de la Recherche Agronomique (INRA), Centre de Coopération Internationale pour la Recherche Agronomique pour le Développement (CIRAD), Paris France, 35p. http://www.gip-ifrai.fr/gip ifrai/activites programmes de 1 ifrai/prospec tive agrimonde.

ISO (International Organization for Standardisation) International Standard 14040 1997. Environmental management. Life cycle assessment. Principles and framework. Geneva: International Organisation for Standardisation.

ISO (International Organization for Standardisation) International Standard 14042, 2000 Environmental management. Life cycle assessment. Life cycle impact assessment. Geneva International Organisation for Standardisation.

Ingrand S., Faye B., 2004. Modelling of herd functioning in France and in tropical areas. To describe, analyse and simulate for understanding, supporting and teaching. Anim. Res., Special Issue, 53, 341-450.

Jollivet M., 1988. Pour une agriculture diversifiée. Ed. L'Harmattan, Collection alternatives rurales. Paris, France, 229p.

Kaimowitz D., Angelsen A., 1998. Economic models of tropical deforestation: Review. CIFOR, Bogor, 139p.

Kerdoncuff M., 2007. Qualification de la participation d'éleveurs à la conception d'un modèle informatique représentant le fonctionnement de leur exploitation : cas de l'élevage bovin laitier à la Réunion. Mémoire de fin d'études d'ingénieur, SupAgro, Montpellier, France, 60p.

Kruska R.L., Reid R.S., Thornton P.K. Henninger N., Kristjanson P.M., 2003. Mapping livestock-oriented agricultural production systems for the developing world. Agric. Syst., 77, 39-63.

Lamine C., Bellon S., 2009 Conversion to organic farming: a multidimensional research object at the crossroads of agricultural and social sciences. A review. Agron. Sustain. Dev., $29,97-112$

Lemery B., 2003. Les agriculteurs dans la fabrique d'une nouvelle agriculture. Sociologie du travail, 9-25.

Lemery B., Ingrand S., Dedieu B., Degrange B., 2005. Agir en situation d'incertitude : le cas des éleveurs de bovins allaitants. Econ. Rurale, 288, 57-69.

Lhoste P., 2002. Les systèmes d'élevage en zones sèches : les conditions de la durabilité 53 ème Réunion Ann. Féd. Eur. Zootech., Le Caire, Egypte, 1-4 septembre, 14p.

Ludovino R.M.R., 2002. Análise da diversidade e da dinâmica da agricultura familiar na Amazônia Oriental - O caso da zona Bragantina. Universidade Técnica de Lisboa, Lisboa, Portugal, 370p.

Mahieu M., Aumont G., 2009. Effects of sheep and cattle alternate grazing on sheep parasitism and production. Trop. Anim. Health Prod., 41, 229-239.

Mahieu M., Archimède H., Fleury J., Mandonnet N., Alexandre G., 2008. Intensive grazing system for small ruminants in the Tropics: The French West Indies experience and perspectives. Small Rum. Res., 77, 195-207.

Mak S., 2001. Continued innovation in a Cambodian rice-based farming system: farmer testing and recombination of new elements, Agric. Syst., 69, 137-149.

Mandonnet N., Tillard E., Faye B., Collin A., Gourdine J.L., Naves M., Bastianelli D., TixierBoichard M., Renaudeau D., 2011. Adaptation des animaux d'élevage aux multiples contraintes des régions chaudes. In : Numéro spécial, Elevage en régions chaudes. Coulon J.B., Lecomte P., Boval M., Perez J.M. (Eds). INRA Prod. Anim., 24, 41-64.

Manoli C., Dedieu B., Ickowicz A., 2010 Livestock farming systems and local development: a review of the multiple dimensions of Territory livestock science. In: Building sustainable rural future: The added value of systems approaches in times of change and uncertainty. Darnhofer I, Grötser M. (Eds). 9ème IFSA Symp., 4-6 July, Vienna, Austria, 2167-2175. www.ifsa.boku.ac.at/

Martin G., Hossard L., Theau J.P., Therond O., Josien E., Cruz P., Rellier J.P., Martin- 
Clouaire R., Duru M., 2009. Characterizing potential flexibility in grassland use. Application to the French Aubrac area. Agron. Sustain. Dev., 29, 381-389.

Martin-Clouaire R., Rellier J.P., 2009. Modelling and simulating work practices in agriculture. Int. J. Metadata, Semantics and Ontologies, 4, 42-53.

Meynard J.M., Aggeri F., Coulon J.B., Habib R., 2006. Recherches sur la conception de systèmes agricoles innovants. Doc INRA, 56p. + annexes.

Mosnier C., Agabriel J., Lherm M., 2009. A dynamic bio-economic model to simulate optimal adjustments of suckler cow farm management to production and market shocks in France. Agric. Syst., 102, 77-88.

Mungkung R., Gheerwala S.H., Bonnet S., Hospido A., Kanyarushoki C., Van der Werf H., Poovarodom N., 2010. Carbon Footprinting and Labelling experiences in Thailand. Thailand-EC Cooperation Facility, Capacity Building of Thai food industries on carbon footprint labelling, 59p.

Mungkung T.R., Udo de Haes H.A., Clift R., 2006. Potential and limitations of Life Cycle Assessment in setting ecolabelling criteria: a case study of Thai shrimp aquaculture product. Int. J. LCA, 11, 55-59.

Nepstad D., Mc Grath D., Alencar A., Barros A.C., Carvalho G., Santilli M., del C. Vera Diaz M., 2002. Frontier governance in Amazonia. Science, 295, 629-631.

Nozière M.O., Moulin C.H., Dedieu B., 2011. The herd, a source of flexibility for livestock farming systems faced with uncertainties. Animal, Accepté,

Piketty M.G., 2003. Mudanças do uso da terra e da floresta e efeito estufa nas frentes pioneiras da Amazônia Brasileira. São Paulo, USP Rapport, $46 \mathrm{p}$.

Poccard-Chapuis R., Thales M., Venturieri A., Piketty M.G., Mertens B., Bastos da Veiga J., Tourrand J.F., 2005. La filière viande : un levier pour contrôler les dynamiques pionnières en Amazonie brésilienne. Cah. Agric., 14, 53-58.

Rey-Valette H., Clément O., Aubin J., Mathé S., Chia E., Legendre M., Caruso D., Mikolasek O., Blancheton J.P., Slembrouck J., Baruthio A., René F., Levang P., Morissens P., Lazard J., 2008. Guide de co-construction d'indicateurs de développement durable en aquaculture. Cirad, Ifremer, INRA, IRD, Université Montpellier 1. Diffusion Cirad-Montpellier, France, 144p.

Röling N., de Jong, F., 1998. Learning: Shifting paradigms in education and extension studies. J. Agric. Educ. Extension, 5, 143-161.

Sauvant D., Baumont R., Faverdin P., 1996. Development of a mechanistic model of intake and chewing activities of sheep. J. Anim. Sci., 74, 2785-2802.
Sciortino M., 2010. Current understanding of gobal climate change and of its possible impacts on agriculture. www.eaap.org/Crete/ Sessions/Plenary Session.html

Scoones I., 1999, Nouvelles orientations du développement pastoral en Afrique. Vivre dans un environnement incertain. Editions Karthala et CTA, Londres, UK, 362p.

Seegers J., Moreau J.C., Béguin E., Guillaumin A., Frappat B., 2006. Attentes des éleveurs laitiers vis-à-vis de leurs conditions de travail et évolution de leurs systèmes d'exploitation, Fourrages, 185, 3-16.

Sere C., Steinfeld H., 1996. World livestock production systems: current status, issues and trends. FAO. Rome, Italie. Anim. Prod. Health Paper, 127.

Spies A., 2003. The sustainability of the pig and poultry industries in Santa Catarina, Brazil : a framework for change. University of Queensland, School of Natural and Rural Systems Management, Brisbane, Australia, 379p.

Suzuki K., Kanameda M., Ogawa T., Nguyen T.T.D., Dang T.T.S., Luu Q.H., Pfeiffer D.U., 2006. Productivity and socioeconomic profile of dairy cattle farmers amongst rural smallholder communities in northern Vietnam. Livest. Sci., 101, 242-250.

Stark F., Alexandre R., Diman C., Fanchone A., Alexandre G., Diman J.L., 2010. Intégration au sein des systèmes de type polyculture élevage en Guadeloupe : première caractérisation. Renc. Rech. Rum., 421-424.

Steinfeld H., Gerber P., Wassenaar T., Castel V., Rosales M., de Haan C., 2006. Livestock's Long Shadow. Environmental Issues and Options, FAO, Rome, 464p.

Steinfeld H., Mooney H., Schneider F., Neville L., 2010. Livestock in a Changing Landscape. Drivers, Consequences, and Responses, Ed. Island Press, Washington D.C., USA, 1, 416p.

Thewis A., Bourbouze A., Compère R., Duplan J.M., Hardouin J., 2005. Manuel de zootechnie comparée Nord-Sud, INRA, Paris, France, 656p.

Vayssières J., 2004. L'appréhension des pratiques décisionnelles d'éleveurs par enquêteimmersion : cas des activités à l'origine de flux d'azote en exploitations bovines laitières à la Réunion. Rapport de stage DEA, INA-PG, Paris, France, 158p.

Vayssières J., Lecomte P., 2007. Modéliser les pratiques décisionnelles et les flux d'azote à l'échelle globale de l'exploitation : cas de l'élevage bovin laitier en contexte tropical insulaire. Renc. Rech. Rum., 45-48.

Vayssières J., Lecomte P., Guerrin F., Bocquier F., Verdet C., 2006. Explaining the diversity of environmental performances according to a typology of farming practices combinations: the case of the dairy cattle breeding in Reunion Island. Proc. $12^{\text {th }}$ RAMIRAN Int. Conf. Technology for recycling of manure and organic residues in a whole-farm perspective. 11-13 September, Aarhus, Denmark, 2, 57-60.

Vayssières J., Bocquier F., Lecomte P., 2009a. GAMEDE: a Global Activity Model for Evaluating the sustainability of Dairy Enterprises. Part II. Interactive simulation of various management strategies with diverse stakeholders. Agric. Syst., 101, 139-151.

Vayssières J., Guerrin F., Paillat J.M., Lecomte P., 2009b. GAMEDE: a Global Activity Model for Evaluating the sustainability of Dairy Enterprises. Part I. Whole-farm dynamic model. Agric. Syst., 101, 128-138.

Vayssières J., Vigne M., Alary V., Lecomte P., 2011. Integrated participatory modelling of actual farms to support policy making on sustainable intensification. Agric. Syst., sous presse.

Vavra M., 1996. Sustainability of animal production systems: An ecological perspective. J. Anim. Sci., 74, 1418-1423.

Vereijken P., 1997. A methodical way of prototyping integrated and arable farming systems in interaction with pilot farms. Eur. J. Agron., 7, 235-250.

Vigne M., Vayssières J., Bochu J.L., Lecomte P., 2009. Energy use efficiency and greenhouse gases emissions of dairy farms of an isolated territory : case of Reunion Island from 2000 to 2007. In: AgSAP Int. Conf., 1012 Mars, Egmond aan Zee, the Netherlands, 430-431.

Vilain L., 2003. La méthode IDEA. Indicateurs de durabilité des exploitations agricoles. Guide d'utilisation, 2ème édition, Editions Educagri, Dijon, France, 100p.

Wane A., Ancey V., Diao-Camara A., Ndiobéne Kâ S., Touré I., 2010. L'économie pastorale face aux incertitudes. Le salariat au Sahel du Sénégal. Cah. Agric., 19, 359-365.

Yung J.M., Zaslavsky J., 1992. Pour une prise en compte des stratégies des producteurs, Documents Systèmes Agraires, Montpellier, France, $72 \mathrm{p}$.

Zébus M.F. Alexandre G., Diman J.L., Despois E., Xandé A., 2004. Activités informelles, normalisation et développement dans l'agriculture caribéenne. Cas de l'élevage porcin en Guadeloupe. Cah. Agric., 13, 263-270.

Zébus M.F., Alexandre G., Diman J.L., Paul J.L., Despois E., Phaëton E., 2005. Diversité des élevages porcins en Guadeloupe, 1 ère évaluation technico-économique. Journ. Rech. Porcine Fr., 37, 407-412. 


\title{
Résumé
}

Les démarches de conception et d'évaluation de systèmes d'élevage innovants en régions chaudes doivent prendre en compte les spécificités de ces systèmes. Outre les caractéristiques des milieux, ces spécificités tiennent à l'importance des fonctions non productives des troupeaux et à la complexité des modes de gestion de l'accès aux ressources (mobilité notamment). Nous discutons les démarches de conception en utilisant les notions de cristallisation, de plasticité et de développement des systèmes innovants et présentons les bases de l'évaluation (environnementale, filière, technico-économique) et ses fonctions dans le processus de conception. Nous illustrons la conception réglée ou innovante appuyée sur de la modélisation à l'aide d'exemples aux Antilles (élevage caprin, polycultureélevage, avec une composante biotechnique forte), à la Réunion (élevage laitier avec une démarche participative de construction du modèle avec les agriculteurs) et en Amazonie (composante décisionnelle incluant les équilibres et interaction entre activités d'élevage et culture - démarche participative avec les acteurs territoriaux). La diversité des cas souligne les enjeux de la conception et de l'évaluation : prise en compte de la diversité des systèmes dans leurs composantes techniques et humaines, participation des acteurs à la démarche, développements biotechniques et clarification des processus décisionnels, réflexion sur des indicateurs adaptés et pertinents pour les éleveurs et les territoires dans lesquels ils s'insèrent.

\begin{abstract}
Design and evaluation of sustainable livestock farming systems in warm regions

The processes for designing and evaluating innovative livestock systems in warm regions must take the specificities of these systems into account. In addition to the characteristics of the surrounding environment, these specific features are due to the non-productive functions of the livestock (savings bank on the hoof) and the complexity of management methods (in particular mobility) and are about who is «at the helm». We discuss approaches to design, with notions of crystallisation, plasticity and development and we present the bases of the evaluation (environmental, chain, technico-economic) and its functions in the design process. We illustrate rule-based or innovative design supported by modelling, using examples in the West Indies (goat farming, mixed crop-livestock farming, with a strong biotechnical component), in La Réunion (dairy farming with a participative approach with farmers for building the model) and in Amazonia (decisional component including balances and interaction between farming and cropping activitiesparti-cipative approach with territorial players). The diversity of cases underlines the stakes involved in design and evaluation: taking account of the diversity of systems in their technical and human components, participation of players in the approach, biotechnical developments and clarification of decisional processes, and thought given to indicators that are appropriate and relevant for livestock farmers.
\end{abstract}

DEDIEU B., AUBIN J., DUTEURTRE G., ALEXANDRE G., VAYSSIERES J., BOMMEL P., FAYE B. Avec la collaboration de MAHIEU M., FANCHONE A., TOURRAND J.-F., ICKOWICZ A., 2011. Conception et évaluation de systèmes d'élevage durables en régions chaudes. In : Numéro spécial, Elevage en régions chaudes. Coulon J.B., Lecomte P., Boval M., Perez J.M. (Eds). INRA Prod. Anim., 24, 113-128. 